University of Rhode Island

DigitalCommons@URI

Open Access Dissertations

2017

\title{
Impact of Multicultural Ethnic Identity on Emerging Adult Alcohol and Marijuana Use
}

Emilie B. Joseph

University of Rhode Island, emilie_joseph@my.uri.edu

Follow this and additional works at: https://digitalcommons.uri.edu/oa_diss

\section{Recommended Citation}

Joseph, Emilie B., "Impact of Multicultural Ethnic Identity on Emerging Adult Alcohol and Marijuana Use" (2017). Open Access Dissertations. Paper 604.

https://digitalcommons.uri.edu/oa_diss/604

This Dissertation is brought to you for free and open access by DigitalCommons@URI. It has been accepted for inclusion in Open Access Dissertations by an authorized administrator of DigitalCommons@URI. For more information, please contact digitalcommons-group@uri.edu. 
IMPACT OF MULTIRACIAL ETHNIC IDENTITY ON EMERGING ADULT

ALCOHOL AND MARIJUANA USE

BY

EMILIE B. JOSEPH

A DISSERTATION SUBMITTED IN PARTIAL FULFILLMENT OF THE

REQUIREMENTS FOR THE DEGREE OF

DOCTOR OF PHILOSOPHY

IN

CLINICAL PSYCHOLOGY

UNIVERSITY OF RHODE ISLAND

2017 


\title{
DOCTOR OF PHILOSOPHY DISSERTATION
}

OF

\author{
EMILIE B. JOSEPH
}

APPROVED:

Dissertation Committee:

Major Professor $\quad$ Paul Florin
Mary Clair-Michaud

Abran Salazar

Nasser H. Zawia

DEAN OF THE GRADUATE SCHOOL

UNIVERSITY OF RHODE ISLAND

2017 


\begin{abstract}
Adults identifying with two or more races represent about 7\% of the U.S. populations (Pew Research Study, 2015). Multiracial individuals are a growing population within the United States with unique needs and experiences that are represented to a limited degree within substance use literature. However, existing research has demonstrated that multiracial individuals are at an increased risk for adverse substance use outcomes that remain largely unexplored (Unger, 2012). While existing literature has explored the impact factors like ethnic identity, neighborhood risk and drug beliefs and attitudes amongst monoracial youth and young adults, limited extensions have been made to their multiracial peers to better understand evidence of their increased risk. With a sample of 281 multiracial emerging adults, the current study sought a better understanding of ethnic identity as a protective factor against marijuana and alcohol use in relation to neighborhood risk and attitudes and beliefs about substance use. The current study supports previous findings that ethnic identity, neighborhood risk, personal disapproval, and perceived risk of alcohol and marijuana relates to substance use outcomes; however, results suggest that ethnic identity may not serve a protective role within this sample. Implications of the findings are further discussed and future directions for this area of research are provided.
\end{abstract}




\section{Acknowledgements}

I haven’t arrived to this point in my academic career alone. I have been blessed with an abundance of people who have given me a shoulder to cry on, an ear to listen, and a space to feel loved and validated. I know that my journey would be very different had it not been for the people challenging, encouraging, and believing in me during this long and difficult process.

To my first major professor, Jasmine Mena, I can’t thank you enough for all the wisdom, advice, and encouragement that you gave me during the four years we worked together. I am constantly in awe of you and cannot put into words the transformative impact you have had on me. I know that I am a better person, personally and professionally, having had the opportunity to be mentored and guided by you. To my current major professor (aka my grand MP), Paul Florin, thank you for taking me under your wing and giving me the chance to continue to thrive as I complete my journey. Even before you assumed this role as my current MP, you took me in and shared your kindness and wisdom. I'm forever thankful for having a space to come to when I needed to be around caring and encouraging people.

I want to also thank my committee members, Abran Salazar and Mary-Clair Michaud. Abran, working with you has challenged me to think differently and I've learned to build confidence in my knowledge. Mary-Clair, thank you for stepping in when I needed you. Your willingness to provide me with support when I was most in need has been invaluable.

To my family, I cannot tell you how much I love and appreciate how much you enrich my life. Mom and Dad, you have stood by me and sacrificed for me and I am 
forever grateful. Packie, Ovi, and Fabi, my heart swells when I think of how much you all mean to me and I can’t begin to tell you how grateful I am for the phone calls, laughs, tears, and hugs. You've always been in my corner and know that I'm always in yours.

To my good friends at URI, we say it all the time but it's true, "I could not have made it through grad school without you!”. Clifton, Crystal, Daphne, Janan, Danielle, Uchenna, April, Brittney P., Brittney, W., and Michael, you all kept me sane when things just got “tew much”. The group chats, the potlucks, the girls' nights, the jokes, advice and vent sessions helped me make it through and I am grateful for the lifelong friendships that I've built with all of you.

To my cohort, Miryam, Dorothy, and Isabel, we have families that choose us and then there's the families we choose. You all are the family that I choose. I've built a special bond with each of you that I will cherish forever. Having spent the last four years holding each other up and pushing each other forwards, I can’t imagine my life without your intelligent, thoughtful and caring presences. I can't wait to see what's in store for each of you and continue to share in the ups and downs that life undoubtedly brings our ways. We have been and continue to be strong together.

Lastly to Mark Wood, I can’t thank you enough for your advice, great jokes, and warm guidance. I wish you could have been here to see all of this. To my grandmother, Frasilia Emilienne Piard, while my heart still breaks when I think of your passing, I can still hear you tell me "fè efò”. I'll never stop trying my best, manman. 


\section{TABLE OF CONTENTS}

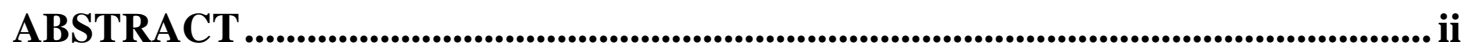

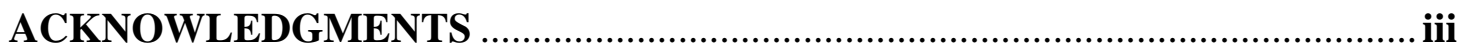

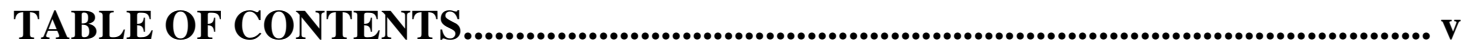

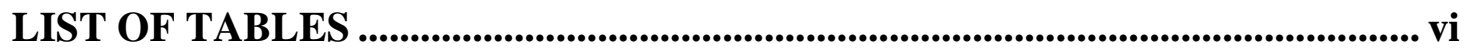

LIST OF FIGURES .......................................................................................... vii

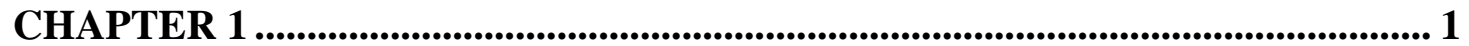

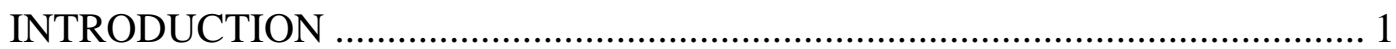

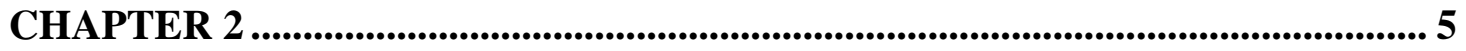

REVIEW OF LITERATURE ................................................................ 5

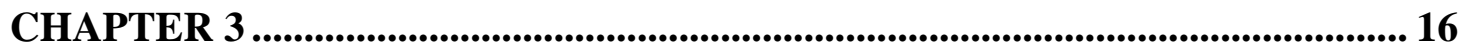

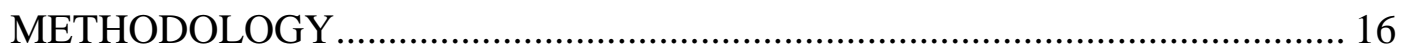

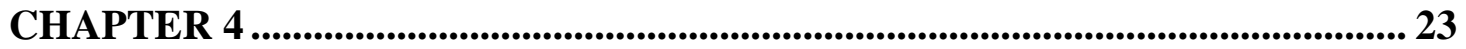

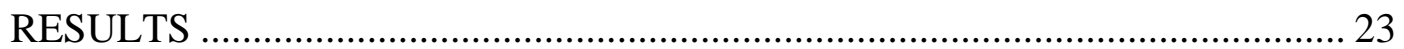

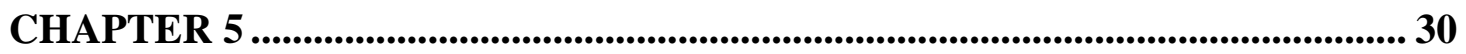

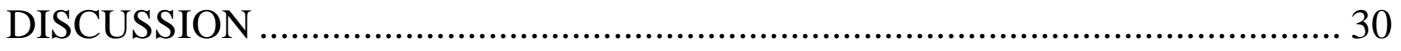

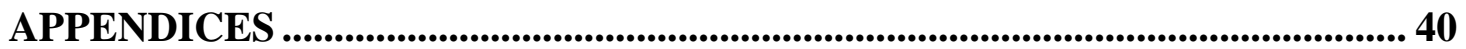

ADDITIONAL INFORMATION REGARDING THE STUDY OF ETHNIC

IDENTITY IN SUBSTANCE USE LITERATURE ........................................... 40

SAMPLE ADVERTISEMENT …………………..................................... 43

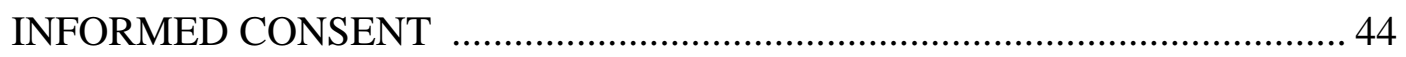

INTENTION TO USE DESCRIPTION AND MEASURES .................................. 46

SURVEY: DEMOGRAPHIC INFORMATION .................................................. 47

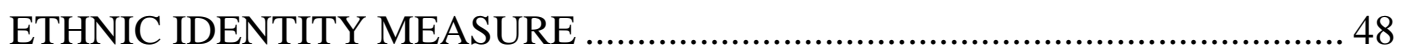

NEIGHBORHOOD RISK MEASURE .......................................................... 50

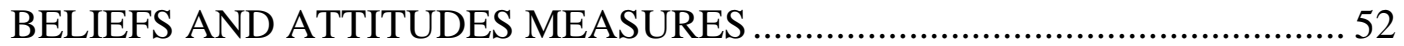

ALCOHOL AND MARIJUANA USE MEASURES............................53

ALCOHOL AND MARIJUANA-RELATED PROBLEMS MEASURES ........55

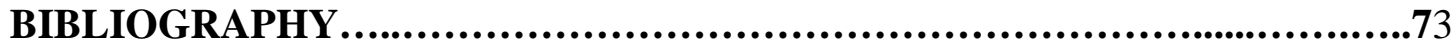




\section{LIST OF TABLES}

TABLE

PAGE

Table 1. Sample Characteristics ............................................................................... 57

Table 2. Self-Identified Racial/Ethnic Groups............................................................. 58

Table 3. Pre-selected Racial/Ethnic Groups (Participants) .......................................... 59

Table 4. Pre-selected Racial/Ethnic Groups (Parent 1).................................................... 60

Table 5. Pre-selected Racial/Ethnic Groups (Parent 2)................................................ 61

Table 6. Cronbach’s Alphas for Scales and Subscales ................................................. 62

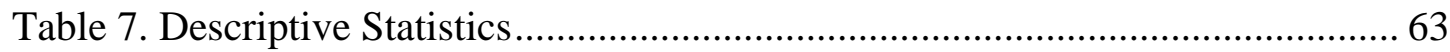

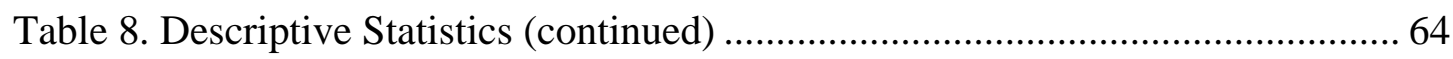

Table 9. Correlations of Scales and Subscales of Interest (Pre-Imputation)................. 65

Table 10. Correlations of the Scales and Subscales of Interest (Post-Imputation) ..... 66

Table 11. Moderation Hierarchical Multiple Regression Results of Marijuana Use

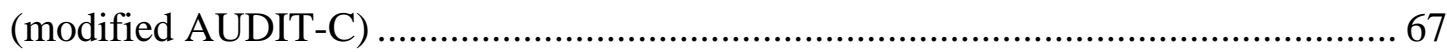

Table 12. Moderation Hierarchical Multiple Regression Results of Alcohol Use

(AUDIT-C)

Table 13. Moderation Hierarchical Multiple Regression Results of Marijuana Use

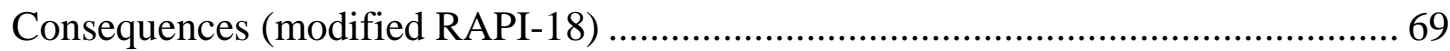

Table 14. Moderation Hierarchical Multiple Regression Results of Alcohol Use

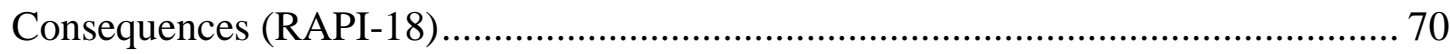




\section{LIST OF FIGURES}

FIGURE

PAGE

Figure 1.1 Model Displaying Expected Moderating Influence of Ethnic Identity. .... 70

Figure 1.2. Model Displaying Mediating Effects of Personal Disapproval and

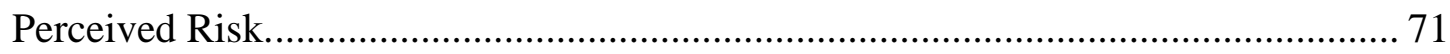




\section{CHAPTER 1}

\section{INTRODUCTION}

Individuals who identify with two or more racial or ethnic groups are part of a rapidly growing population, but have been largely ignored in ethnic identity and substance use research in the past (Chao \& Otsuki-Clutter, 2011; Shih \& Sanchez, 2009; Unger, 2012). Recent changes in the U.S. Census in 2000 that allow individuals to self-identify with multiple racial or ethnic backgrounds have brought increased attention to this population (Shih \& Sanchez, 2009; Unger, 2012) and have encouraged the use of the self-identification method in more recent large scale studies such as the 2001 California Health Interview (Chavez \& Sanchez, 2010). Recent studies have indicated that self-identified multiracial individuals are at higher risk for certain substance use and other adverse behavioral health outcomes (i.e. marijuana use, tobacco use, substance abuse, and suicide) in comparison to monoracial individuals (Chavez \& Sanchez, 2010; Choi, Harachi, Gillmore, \& Catalano, 2006a; Jackson \& LeCroy, 2009; Sakai, Wang, \& Price, 2010; Shih \& Sanchez, 2005; Udry, Li, \& Hendrickson-Smith, 2003).

The prevalence rates of alcohol use and misuse among emerging adults demonstrate that problematic drinking continues to be a major concern for this population. As of 2012, 39.8\% of 18-25 year-old men and women across all racial and ethnic groups engaged in binge drinking (Substance Abuse and Mental Health Services Administration [SAMHSA], 2012). Over one-third of college students, and their non-college peers, engaged in heavy drinking (i.e., consuming five or more 
drinking at least once in two weeks) (Johnston, O’Malley, Bachman, \& Schulenberg, 2013b). In addition to heavy drinking, $40 \%$ of college students report drinking to the point of getting drunk within the last 30 days in comparison to $36 \%$ of their noncollege peers (Johnston et al., 2013b).

While heavy drinking can be problematic in and of itself, problematic alcohol use has also been found to impact physical and psychological well-being in the long term (White \& Jackson, 2005; Rehm, 2011). These consequences include fatal and non-fatal injuries, overdosing, physical and sexual assault, and sexually transmitted diseases (White \& Jackson, 2005). Problematic use is especially concerning for emerging adults because it can negatively impact attainment of traditional adult roles (White \& Jackson, 2005). Consequences such as academic and career-related failures and unintended pregnancies can occur as result of alcohol misuse in this population (White \& Jackson, 2005). Further legal complications involving violence and crime, vandalism, and disrupting the community are other potential consequences that have been observed within this population (White \& Jackson, 2005). In summary, existing research has demonstrated that alcohol use is associated with social, physical, and mental costs to the individual user and their larger social environment (Rehm, 2011).

Despite being identified as a group facing higher risk for substance use, limited studies of alcohol use and misuse have focused on multiracial populations. Prevalence rates within this population support growing concern for youth and young adults that identify with two or more races. According to SAMHSA (2012), almost 52\% Multiracial individuals ages 12 and older report alcohol use within the past 30 days; this is second only to White individuals (57.4\%) of the same age. Additionally, 
lifetime substance use rates, a measure that taps into whether an individual has ever tried a substance over the course of their live, are higher among multiracial adolescents across four categories (alcohol, marijuana, inhalants, and tobacco) in comparison to their monoracial peers (Jackson \& LeCroy, 2009). For example, multiracial Caucasian (about 78\%), Native Hawaiian (over 80\%), and Asian youth (ranging from 70-80\% across 6 different ethnic groups) have reported higher prevalence of lifetime alcohol use than their monoracial peers (Sakai et al., 2010). Limited research has explored the consequences associated with problematic alcohol use in this population, but available research suggests that multiracial youth report higher rates of problem behaviors such as violent behavior than their monoracial peers (Choi et al., 2006b).

Marijuana use, and the associated consequences, is also an important area of study within the emerging adult population. Over one third of college students and one third of their non-college peers engage in marijuana use annually (Johnston et al., 2013b). On a daily basis $9.4 \%$ of non-college young adults engage in marijuana use and about half that percentage (4.7\%) of college students engage in daily use (Johnston et al., 2013b). Cohort effects will lead to an increase in rates of marijuana use among college students and other young adults in the coming years (Johnston et al., 2013b). This will occur because recent increases in teenage marijuana use are expected to translate into higher rates of use in young adults as this cohort grows older and generational replacement occurs (Johnston et al., 2013b).

As marijuana use increases in this population it will be important to be mindful of some of the negative consequences experienced by young adults as a result of 
marijuana use. College students have been found to drive under the influence, miss school or work, fight, neglect their responsibilities, and experience disruptions in their academic work (Simon, Correia, Carey, \& Borsari, 1998). When we take a closer look at multiracial youth, individuals who identify with two or more races report the highest levels of illicit drug use (SAMHSA, 2012). When compared to some of their monoracial peers, multiracial youth have statistically significant higher rates of substance use (rate= 1.29) than their white peers (rate $=1.19)($ Choi, Harachi, \& Catalano, 2006b). Additionally, White and Asian American monoracial youth are $48 \%$ and $76 \%$, respectively, less likely to initiate marijuana use than their multiracial peers (Choi et al., 2006b). Just as with alcohol use, multiracial youth exhibit problem behaviors (e.g., fighting) at higher rates than their monoracial peers who were over 50\% less likely to engage in violent or threatening behaviors (Choi et al., 2006a). 


\section{CHAPTER 2}

\section{REVIEW OF LITERATURE}

\section{Models of Adolescent Substance Use}

Previous research has largely focused on monoracial youth and young adults and there is a limited understanding of pathways to alcohol and marijuana use among multiracial young adults (Unger, 2012). While research has established that multiracial youth are at higher risk, we do not yet understand what contributes to that risk or how to protect this population from that risk. Existing models of risk and protection factor research conducted with monoracial populations provide some insight into what pathways may be at work with multiracial youth and young adults.

Many factors have been found to interact and cumulatively contribute to the risk for substance use outcomes (Hawkins, Catalano, \& Miller, 1992). These risk factors generally fall within the following two groups: contextual risk factors and individual and interpersonal risk factors (Hawkins et al., 1992). Economic deprivation and neighborhood disorganization are examples of contextual factors and favorable attitudes towards drug use are an example of individual factors (Hawkins et al., 1992). This general model has been instrumental in identifying the diverse risk factors that converge to produce various outcomes in relation to substance use; however, further research is needed to identify and understand the role of specific protective factors to extend the model. 
Following this general model of risk and protection, research on racial minorities has been able to uncover specific risk factors that differentially impact certain racial and ethnic groups. Contextual factors related to the neighborhood context frequently appear in research exploring risk factors with monoracial populations. For example, negative perceptions of neighborhood have been found to be associated with higher drug use for African American adolescents (Lambert, Brown, Philips, \& Ialongo, 2004). In contrast, African Americans, Asians, and Black Caribbean youth living in affluent neighborhoods have an increased risk of meeting criteria for a substance use disorder in the past year than their other monoracial peers (Molina, Alegría, \& Chen, 2012). For Mexican American youth, residential instability has been found to have a significant impact on cigarette use (Kulis, Marsiglia, Sicotte, \& Nieri, 2007). For example, acculturation gaps between Hispanic immigrant parents and their adolescent children, contribute to engagement in substance use and other risky behaviors (Schwartz et al., 2012). Research into individual and interpersonal factors has highlighted the role of attitudes and beliefs such as personal disapproval and perceived risk. We know that positive alcohol expectancies and declines in perceived risk and personal disapproval are associated with increases in use (Brenner, Bauermeister, \& Zimmerman, 2011; Chartier, Hesselbrock, \& Hesselbrock, 2009; Johnston et al., 2013b SAMHSA, 2012).

Exploration of the risk and protection model has also resulted in the identification of protective factors that may be especially meaningful for racial minorities. Factors related to ethnicity, race, culture, nativity status, and ethnic identity differentially impact specific groups (Kulis et al., 2007; Molina et al., 2012; 
Ndiaye, Hecht, Wagstaff, \& Elek, 2009). For example, systematic hostility and poor support within their receiving community negatively impact youth outcomes among immigrant minority populations (Schwartz et al., 2012). In contrast, Latino youth living in neighborhood with higher concentrations of other Latinos and Latino immigrants have been found to have lower risk of meeting criteria for alcohol use disorder (Molina et al., 2012). Similarly, Mexican American youth living in neighborhoods with a higher immigrant composition had lower rates of substance use (Kulis et al., 2007). Ethnic identity has been found to have weak to no protective impact for some racial groups (Baldwin, Brown, Wayment, Nez, \& Belsford, 2011) and have even been found to promote a higher frequency of heavy drinking when ethnic identity is stronger (e.g., with Latino males) (Zamboanga, Raffaelli, \& Horton, 2006). On the other hand, some monoracial groups experience decreases in heavy drinking and regular marijuana use (Love et al., 2006) and lower intention to use drugs (Ndiaye et al., 2009) as a result of strong ethnic identity. For example, stronger ethnic identification has been shown to protect African American women from the harmful impact of illicit drug use (Steven-Watkins, Perry, Harp, \& Oser, 2012). Conversely, low ethnic identity has been associated with nicotine dependence (Brook, Duan, Brook, \& Ning, 2007) and adult smoking (Brook, Zhang, Finch, \& Brook, 2010). Research has demonstrated that discrimination experiences are associated with higher levels of nicotine dependence (Kendzor et al., 2013). It is important to note that this relationship between ethnic identity and nicotine use can be related to discrimination within ethnic minority populations (Kendzor et al., 2013). 
Working within the framework of risk and protection factors established by the general model and previous research, the current study will review specific research related to neighborhood risk, ethnic identity, personal disapproval, and perceived risk. Brief explanations of the concepts and relevant research will be discussed and extensions of this research and applications to multiracial emerging adult populations will be discussed.

\section{Further Discussion of Risk and Protective Factors}

\section{Neighborhood Risk}

Neighborhoods are defined as “complex systems of resources which can facilitate or inhibit positive health outcomes” (Bernard et al., 2007). Neighborhood risk is therefore defined as "patterns of health inequalities" determined by the number of, amount of and access to resources within these contexts (Bernard et al., 2007). Essentially, neighborhood risk assessed using the number of health inequalities that result from limited access to important resources in the immediate environment. Resources fall within the following five categories: physical features of the environment, quality of services, sociocultural features, presence of supports for health lifestyles, and the area’s reputation (Bernard et al., 2007).

The most commonly used measure of neighborhood risk is neighborhood disadvantage (i.e., a combination of percentages of those living below the poverty line, households led by females, families receiving public assistance, and male unemployment rates) (Boardman, Finch, Ellison, Williams, \& Jackson, 2001). The research documenting the relationship between neighborhood disadvantage and substance use is mixed. Some studies focusing on neighborhood disadvantage and 
neighborhood's socioeconomic climate have found that higher neighborhood disadvantage or lower economic stability is associated with greater reports of drug use in the past year (Boardman et al., 2001; Chauhan \& Widom, 2012), higher cigarette and tobacco use (Businelle et al., 2010; Karriker-Jaffer, 2013), and higher rates of drinking (Jones-Webb \& Karriker, 2013) and regular marijuana use (Karriker-Jaffer, 2013). Other studies have found no direct relations between adolescent substance use and neighborhood disadvantage (Brenner et al., 2011) and no significant association between neighborhood disadvantage and alcohol (Stockdale et al., 2007; Fagan, Wright, \& Pinchevsky, 2012) and marijuana use (Sunder, Grady, \& Wu, 2007; Fagan, Wright, \& Pinchevsky, 2012). Brenner et al. (2011) suggested that mixed results have been found because measures of neighborhood disadvantage do not adequately capture meaningful neighborhood factors that influence youth health and behavior. Other neighborhood factors that more adequately capture meaningful influences on youth health and behavior are often studied individually or in isolation. These risk factors include neighborhood disorder, neighborhood composition, attachment to neighborhood, and social factors (e.g., control, safety, violence). However, studying these factors separately ignores the assessment of cumulative risk that is associated with multiple risk factors.

Choi et al. (2016a) suggested that neighborhood characteristics are especially important for multiracial youth. Multiracial youth report higher rates of substance use than their monoracial peers but they are also more likely to report living in neighborhoods with higher risk (Choi et al., 2006a). Lack of attachment and safety in neighborhood was associated with higher rates of substance use among multiracial 
youth (Choi et al., 2006a). While this study is one of few that sought to understand the unique experiences of multiracial youth in comparison to their monoracial peers, their assessment of neighborhood characteristics was limited (i.e., they only looked at three areas of risk) and did not consider the cumulative risk of the neighborhood characteristics studied.

\section{Ethnic Identity}

Ethnicity and race categories have been widely used to assess group differences within substance use literature; however, the use of constructs, such as ethnic identity, that are more individually meaningful and tap into the dynamic nature of cultural identity is gaining more support (Unger, 2012). Ethnicity is most commonly determined by parents' heritage and therefore this label is assigned and is considered to be an arguably objective way of labeling individuals (Phinney, 1992). On the other hand, ethnic identity involves self-identification (i.e., choosing an ethnic group for oneself) and it is thought to be based on subjective orientation to one or more ethnic or cultural groups (Phinney, 1992).

Much of what we know about ethnic identity, within the context of substance use literature, was gleaned from monoracial youth populations. For example, strong ethnic identity has been found to protect African American youth from heavy alcohol use (Nasim, Belgrave, Jagers, Wilson, \& Owens, 2007) and African American young adults from stated willingness to use and actual use of alcohol (Stock et al., 2013). When placed within the context of neighborhood risk, ethnic identity has been found to moderate the relation between neighborhood risk and lower intentions to use drugs in the future and greater disapproval of drug use (Corneille \& Belgrave, 2007) 
while social ties within the neighborhood appear to buffer the impact of neighborhood disorder (Gapen et al., 2011).

Despite the extensive literature available on ethnic identity with monoracial populations, we know very little about ethnic identity within multiracial youth. Chao \& Otsuki-Clutter (2011) found that multiethnic and multiracial adolescents are largely absent from studies of racial and ethnic identity and substance use. Multiethnic and multiracial young adults are also largely ignored in this area of research. The research that is available is limited and focuses largely on comparing rates of substance use between multiracial and monoracial individuals. Although this research has been instrumental in drawing attention to this group because we know that multiracial adolescents are more likely than some of their monoracial peers to initiate alcohol use and report rates of substance use that is similar to other high risk groups (e.g., Native Americans and European Americans) (Chavez \& Sanchez, 2010). However, the source of that risk is still unknown (Udry et al., 2003). Existing theories suggest that ethnic identity may be important for multiracial individuals for the maintenance of a bicultural or integrated ethnic identity (Nagoshi, Marsiglia, Parsai, \& Castro, 2011). Available research has also suggested that ethnic identity development can positively (e.g., sense of comfort associated with addressing identity issues) and negatively (e.g., negative experience associated with defining racial identity) impact multiracial youth as they engage in developmental processes (Shih \& Sanchez, 2005). Additionally, research has found that experiences focused on race and multiculturalism can have an enduring impact on how individuals psychologically manage multiple racial identities (Cheng \& Lee, 2009). In contrast, other research has found limited evidence to support 
the idea that multiracial individuals suffer from negative outcomes related to identity development and theorizes that multiracial individuals may have a different relationship with their identity development process than their monoracial peers (Shih \& Sanchez, 2005). In sum, while this research has demonstrated the importance of further study within multiracial populations, we know very little about the ethnic identity development of multiracial youth and even less about its implications for psychological and behavioral health outcomes. (Additional information about ethnic identity research can be found in Appendix A).

\section{Drug Attitudes and Beliefs, and Their Relationship to Neighborhood Characteristics and Ethnic Identity}

While there are a multitude of constructs that fall within the categories of drug related beliefs and attitudes, this study will focus specifically on perceived risk and personal disapproval. Perceived risk is defined as "seeing great risk associated with substance use” (Johnston et al., 2012). Personal disapproval is defined as the level of “disapproval associated with use of a specific substance” (Johnston et al., 2012). As

mentioned previously, positive alcohol expectancies and declines in perceived risk and personal disapproval have been consistently associated with increases in use (Brenner, Bauermeister, \& Zimmerman, 2011; Chartier, Hesselbrock, \& Hesselbrock, 2009; Johnston et al., 2013b SAMHSA, 2012).

Beliefs and attitudes have also been linked to drug use through their association with neighborhood characteristics and ethnic identity. Neighborhood drinking norms (e.g., permissive norms related to drunkenness) have been found to influence drinking behavior (e.g., greater odds of moderate and binge drinking) 
presumably by lowering perceived risk and personal disapproval (Ahern, Galea, Hubbard, Midanik, \& Sims, 2008). Additionally, individuals that endorse norms that encourage drunkenness have been found to consume larger amounts of alcohol and experience more alcohol-related consequences as a result (Jones-Webb \& KarrikerJaffe, 2013). Conversely, other studies have found that youth reporting higher neighborhood risk reported greater disapproval and higher perceived risk, with lower drug use (Corneille \& Belgrave, 2007; Lambert et al., 2004). Available models suggest that drug beliefs mediate the relationship between neighborhood characteristics and substance use outcomes (Lambert et al., 2004).

When looking at ethnic identity, low ethnic identity has been associated with more positive substance use expectancies and greater intentions to engage in substance use in the future (Ndiaye et al., 2009). Conversely, stronger racial identity among Black young adults has been associated with low perceptions of friends' use, less favorable perceptions of substance users, and lower levels of willingness to use leading to lower actual use (Stock, Gibbons, Walsh, \& Gerrard, 2011). Students reporting strong ethnic identity also report less approval of peers who use drugs and more confidence in their abilities to refuse drugs (Ndiaye et al., 2009). Many of these studies have focused on monoracial adolescents and intentions to use drugs in the future as opposed to measuring current or past use. Available literature with multiracial youth has found the views of substance use are significantly related to patterns of binge drinking and illicit drug use (Chen, Balan, \& Price, 2012) but we know very little about the impact of beliefs and attitudes within multiracial 
populations and the limited research that does exist focuses largely on adolescents, not emerging young adults which is the focus of the present study.

\section{The Present Study}

The current study seeks to extend the literature on multiracial youth to young adults, develop a better understanding of ethnic identity within this population and its role as a protective factors, apply a more comprehensive measure of neighborhood risk that include consideration of cumulative risk, and provide statistical support for the role of perceived risk and personal disapproval as mediating factors between neighborhood risk and substance use outcomes.

The current study explored the following hypotheses in order to address the limitations existing in current literature regarding substance use outcomes in the multiracial young adult population:

H1. It was predicted that strong positive relations between neighborhood risk and marijuana and alcohol use and alcohol and marijuana-related problems would be found.

H2. It was predicted that strong negative relations between strength of ethnic identity of multiracial individuals and marijuana and alcohol use and alcohol and marijuana-related problems would be found.

H3. It was predicted that strong negative relations between perceived risk and personal disapproval and marijuana and alcohol use and alcohol and marijuana-related problems would be found. 
H4. It was predicted that strength of ethnic identity of multiracial individuals would moderate the impact of neighborhood risk, perceived risk, and personal disapproval on marijuana and alcohol use and alcohol and marijuana-related problems.

H5. It was predicted that perceived risk and personal disapproval would mediate the relationship between neighborhood risk and marijuana and alcohol use.

Limited extensions have been made to multiracial young adult populations who are at similar or greater risk for alcohol and marijuana use than some of their monoracial peers. While available literature has played a key role in drawing attention to this population, we essentially have substance use rates for multiracial youth but limited research discussing the etiology of substance use in this population and more research is needed. The current study adds to the current body of literature by elaborating on the influence of the variables of interest in a population that has been afforded little attention. 


\section{CHAPTER 3}

\section{METHODOLOGY}

\section{Participants}

A total of 1,316 individuals accessed the online survey. After applying study requirements (identify with 2 or more ethnic groups and young adults aged 18-29) and excluding cases with excessive missing data/incomplete participation, 281 cases were used in the analysis. A summary of participant characteristics can be found in Table 1. The average age of participants was 24.30 years old $(S D=3.14$, range: $18-29)$. Of the total participants, $87.2 \%$ reported identifying with two ethnic groups $(n=245)$. Additionally, 30 participants reported identifying with three ethnic groups and six participants reported identifying with four or five ethnic groups. Participants selfidentified with 71 ethnic groups (Table 2). When asked to identify according to preselected racial/ethnic labels, the highest number of race categories identified by individuals in the sample was reduced to four in comparison to five when selfidentified (Table 3). The highest number of racial/ethnic groups for parents endorsed from a preselected list of racial/ethnic groups was four (Table 4 \& Table 5).

Participants identified with the following sex and gender identities: female ( $n=$ 195), male ( $n=77)$, queergender $(n=3)$, transgender $(n=3)$, and self-identified $(n=$

3). Participants identified with the following sexual identities: heterosexual $(n=222)$, lesbian ( $n=6)$, gay $(n=10)$, bisexual $(n=32)$, queer $(n=4)$, self-identify $(n=7)$. Over half of the participants (69.4\%) identified as having at least one year of college 
$(n=195)$. Additionally, 21 participants endorsed having a graduate level education and 63 participants had a high school level education or attended a training/vocational school. Of the total participants, $65.5 \%$ identified as single and never married ( $n=$ 184). The remaining participant identified as follows: divorced/separated $(n=8)$, married $(n=72)$, and unmarried partners $(n=16)$.

\section{Procedure}

Participants were recruited using an advertisement (Appendix B) distributed via departmental and university mailing lists of multiple Rhode Island universities. Participants were also recruited via CINT, a targeted research recruitment agency. Snowball sampling, where participants refer individuals whom they know fit the study criteria, was also used by encouraging participants who completed the survey to share it with other at the end of the survey. SurveyMonkey was used to administer the questionnaire online. Participants were assured anonymity (i.e., no identifying information, such as IP addresses, were collected).

Participants were asked to answer a screening question used to determine if participants identified with two or more racial or ethnic groups and another question to ensure participants were over 18. Those who met the inclusion criteria were asked to read the Informed Consent Form (Appendix C) for the study. Those who agreed to participate were asked to answer a series of questions regarding their neighborhood, ethnic identity, beliefs and attitudes regarding marijuana and alcohol use and past drug use. Questions regarding intentions to use drugs in the future were asked in order to conduct exploratory analysis (Appendix D). Participants were provided with an 
opportunity to win a $\$ 50$ Amazon gift card; however, no participants elected to provide their email address for awarding of the gift card after completing the survey. Instrumentation/Measures

Demographic. Sex \& gender, age, education, sexual orientation and marital status were collected via a demographic survey (Appendix E).

Ethnic identity. The Multigroup Ethnic Identity Measure - Revised (MEIMR) (Phinney \& Ong, 2007) was administered ( $\alpha=0.81)$. The measure is composed of six items split into two subscales which measure exploration $(\alpha=0.76)$ and commitment ( $\alpha=0.78$ ) (Appendix F). Item examples are as follows: "I have often talked to other people in order to learn more about my ethnic group" and "I feel a strong attachment towards my own ethnic group”. Items were preceded by an open response question that asks respondents to self-identity with an ethnic group.

Responses are measured on a 4-point scale (strongly disagree - 1 to strongly agree 4). Participants were asked to complete the MEIM-R separately for each of their selfidentified ethnic groups. The measure concluded with a list of ethnic groups, as listed on the U.S. Census, that asked respondents to identify their own and their parents' ethnic backgrounds.

Participants received a score for each ethnic group that they listed as part of their ethnic identity. This score was calculated by taking the mean of the items of the scale as a whole (the final range of mean scores is 1 - 4). Those mean scores were combined (for each participant) then divided by the maximum possible score for that participant group (the range of possible summed mean scores is 2 - 20) and standardized by multiplying each score by the constant of 100 (the range of possible 
standardized scores is 25 - 100). For example, if a participant, with two ethnic identities, obtains a mean score of 3 per scale, the final summed mean score is 6 . This final summed mean score is divided by 8 (the highest summed mean score that can be obtained by a participant reporting two ethnic identities) then multiplied by 100 to standardize the score (i.e., resulting in a standardized score of 75).

Standardization of ethnic identity strength scores was required in order to adequately compare scores across each of the aforementioned participant groups. Without standardization, comparison across groups would not be possible (i.e., individuals with two ethnic identities could not be compared to individuals with three ethnic identities and so forth). In order to adequately compare across groups, while accounting for the differences in the number of ethnic groups reported, a constant is used to center the scores and spread them around a common mean to better understand how the scores are related to one another despite coming from uniquely different groups. For this study the Cronbach’s alpha for the six item MEIM-R measure was 0.83 (Table 6).

Neighborhood risk. Participants were prompted to answer questions in this section about the neighborhood in which they grew up. Participants were asked to indicate how long they lived in that neighborhood. Neighborhood Development Assets (NDA) is a measure of resources (i.e. security or structured extracurricular activities) present in the neighborhood that promote positive youth development (Oliva et al., 2012). In the current study, neighborhood risk is defined by an absence of or decreased presence of neighborhood developmental assets. NDA is a 22 item measure $(\alpha=0.93)$ composed of the following four subscales: support \& 
empowerment ( $\alpha=0.91$ ), attachment to neighborhood ( $\alpha=0.91)$, security $(\alpha=0.87$ ), social control $(\alpha=0.85)$, and youth activities $(\alpha=0.80)$. Responses are measured on a 7 point scale (strongly disagree -1 to strongly agree -7 ). Example items include the following: "People in my neighborhood commit crimes and engage in other delinquent activities” and "I identify with my neighborhood”. Item scores on the security subscale were reverse coded. Scores were generated by summing the items for each subscale (score range 6-42 for Support \& Empowerment subscale and score range 428 for remaining subscales) and the overall scale (score range 22 - 154); higher scores indicate the presence of more resources which corresponds with lower neighborhood risk. NDA was validated on a monoracial adolescent population therefore alpha coefficients will be analyzed for the proposed study (Appendix G). For this study the Cronbach’s alpha for the 22 item NDA measure was 0.91 (Table 6).

Perceived risk. Three items taken from the 2011 Monitoring the Future Study (Johnston, O’Malley, Bachman, \& Schulenberg, 2013a) were used to assess perceived risk of marijuana use and four items from the same study will be used to assess perceived risk of alcohol use. Reliability data are not provided by the original study; however, the current study yielded strong alpha coefficients for perceived risk of marijuana use $(\alpha=0.91)$ and perceived risk of alcohol use $(\alpha=0.72)$ (Table 6). Participants rated each of these items using the following categories: no risk (0), slight risk (1), moderate risk (2), and great risk (3). Examples of items are as follows: "try one or two drinks of an alcoholic beverage (beer, wine, or liquor)” and "try marijuana once or twice”. Items were scored on a scale from zero to three with total scores ranging from zero to nine. Johnston et al. (2013a) offered a fifth rating category 
indicating that the drug was unfamiliar but this option was excluded in the current study as that was included to account for very young participants who may not have been exposed to some of the drugs in the Monitoring for the Future study. (Appendix H)

Personal disapproval. Three items taken from the 2011 Monitoring the Future Study (Johnston et al., 2013a) were used to assess personal disapproval of marijuana use and four items from the same study will be used to assess personal disapproval of alcohol use. Reliability data are not provided by the original study; however, the current study yielded strong alpha coefficients for personal disapproval of marijuana use $(\alpha=0.87)$ and personal disapproval of alcohol use $(\alpha=0.73)$ (Table 6). Participants rated each of these items using the following categories: don't disapprove (0), disapprove (1), and strongly disapprove (2). Examples of items are as follows: "trying one or two drinks of an alcoholic beverage (beer, wine, or liquor)" and "trying marijuana once or twice". Items were scored on a scale from zero to three with total scores ranging from zero to nine. A fourth category indicating that the drug is unfamiliar was also be exclude from rating options for this measure. (Appendix H).

Alcohol and marijuana use. Alcohol use was measured using the first three items of the Alcohol Use Disorders Identification Test (AUDIT) (Babor, HigginsBiddle, Saunders, \& Monteiro, 2001) as suggested by Siatz (2005) for the measurement of alcohol consumption (Appendix I). In order to measure marijuana consumption, the first three questions of the AUDIT were adapted to specifically ask about marijuana use (Appendix I). Items included the following: "How often do you have a drink containing alcohol” and "How often do you use marijuana”. The third 
question of the AUDIT was modified according to the definition of heavy marijuana use (i.e., seven or more time in one week) provided by Block \& Ghoneim (1993). A total score was generated by summing the scores of all three questions and scores range from zero to twelve. The Cronbach's alpha for the AUDIT-C was 0.77 and for the modified AUDIT-C was 0.85 (Table 6). Six items measuring intentions to use marijuana and alcohol in the future from the 2011 Monitoring the Future Study (Appendix D) also yielded strong alpha coefficients: Marijuana use intention $(\alpha=$ 0.97) and alcohol use intentions $(\alpha=0.95)$ (Table 6).

Alcohol and marijuana-related problems. The short form of the Rutgers Alcohol Problems Index (RAPI-18) will be used to assess problem drinking (White \& Labouvie, 2000). The RAPI-18 is a short 18 item self-administered screening tool that focuses on the occurrence of specific problems related to problematic alcohol use. The RAPI has been adapted to assess marijuana-related problems (Simons, Correia, Carey \& Borsari, 1998). An adapted form of the RAPI-18 will be used to assess problems related to problematic marijuana use. Items, for both scales, included the following: "Got into fights with other people (friends, relatives, and strangers)” and “Neglected your responsibilities”. Items were rated on a scale from zero to three and the total score ranges from zero to fifty-four. For the current study, the Cronbach’s alphas for each 18 item substance use consequences measure are as follows: Alcohol use consequences $(\alpha=0.97)$ and marijuana use consequences $(\alpha=0.98)$ (Table 6). 


\section{CHAPTER 4}

\section{RESULTS}

\section{Preliminary Analysis}

Assumptions of normality, linearity, multicollinearity and homoscedasticity were assessed. Tables 7 and 8 display means, variability, skewness, and kurtosis of the following scales of interest: MEIM-R, NDA, perceived risk, personal disapproval, AUDIT-C. modified AUDIT-C, RAPI-18 and modified RAPI-18. All skewness and kurtosis values fell within the acceptable ranges of -1 and +1 and -1.5 and +2.0 , respectively. Scatterplots were used to assess normality.

Multicollinearity was explored by assessing Pearson’s correlations among the variables of interest (Table 9). Significant correlations were found between ethnic identity and neighborhood risk $(r=0.25, p<0.001)$, marijuana use $(r=0.14, p<$ 0.05), marijuana use consequences ( $r=0.17, p<0.05$ ), and alcohol use consequences ( $r=0.12, p<0.05)$. Significant correlations were found between neighborhood risk and marijuana use $(r=-0.14, p<0.05)$. Significant correlations were found between perceived risk of alcohol use and alcohol use $(r=-0.22, p<0.001)$. Significant correlations between personal disapproval of marijuana use and marijuana $(r=-0.19$, $p<0.05)$ and alcohol $(r=-0.18, p<0.05)$ use were found. Significant correlations were found between personal disapproval of alcohol use and alcohol use $(r=-0.24, p$ $<0.001$ ). All significant correlations fell below 0.70; therefore, concerns about multicollinearity were eliminated. 
ANOVAs were conducted to assess potential group differences on the following variables of interest: ethnic identity scores, neighborhood risk, perceived risk of marijuana use, perceived risk of alcohol use, personal disapproval of marijuana use, personal disapproval of alcohol use, marijuana use, alcohol use, marijuana use consequences, and alcohol use consequences. In the exploration of gender differences, Leven’s Test of Homogeneity only rendered nonsignificant (interpretable) results for neighborhood risk, $F(4,276)=0.27, p=.09$, ethnic identity $F(4,265)=$ 0.69, $p=0.60$, perceived risk of marijuana $F(4,276)=1.87, p=0.12$ and alcohol $F(4$, $276)=1.74, p=0.14$, and personal disapproval of marijuana $F(4,276)=1.37, p=.24$. However, ANOVA results suggest significant gender differences only on perceived risk of marijuana, $F(4,276)=3.20, p<0.05$ and alcohol, $F(4,276)=2.80, p<0.05$. A Tukey post hoc test revealed that female participants reported significantly higher levels of perceived risk of marijuana use $(0.32 \pm 2.93 \mathrm{~min}, \mathrm{p}=0.04)$ and perceived risk of alcohol use $(0.31 \pm 2.53 \mathrm{~min}, \mathrm{p}=0.02)$ than male participants. There were no other significant differences between participants along the other sex and gender categories reported.

An exploration of potential group differences based on number of ethnic identities reported an ANOVA was conducted. Although Levene’s Test of Homogeneity resulted in nonsignificant results for all aforementioned variables except personal disapproval of marijuana use, the ANOVA results demonstrated no significant differences between groups by number of ethnic groups reported along any of the variables of interest. When focusing on education level group differences, Levene's Test of Homogeneity resulted in nonsignificant results for all 
aforementioned variables except neighborhood risk, marijuana use, and marijuana use consequences. However, the ANOVA results demonstrated no significant difference between groups by education level along any of the variables of interest.

Analysis of the general linear model assumptions demonstrated that all statistical assumptions were met allowing use of regression analysis of this data. The data was analyzed for missing data. The Little’s Missing Completely at Random (MCAR) Test was not significant $(\chi 2=4975.12, \mathrm{df}=5362, p=1.00)$ indicating that data was missing completely at random. Multiple imputation using Estimation Maximization (EM) at the item level was conducted to account for the missing data and pooled or averaged (i.e., imputed) statistics are provided for the main analyses.

\section{Main Analysis}

Hypotheses one through three were evaluated via correlation analyses (Table 10). This correlation data included imputed data. Hypothesis one, which predicted significant positive relationships between neighborhood risk between alcohol and marijuana use and consequences, was partially supported. A significant negative relationship between neighborhood resources (measure of neighborhood risk) and marijuana use ( $r=-0.13, p<0.05)$ was found. Hypothesis two, which predicted a strong negative relationship between ethnic identity and marijuana and alcohol use and consequences, was also partially supported. Significant positive relationships between strength of ethnic identity and marijuana use $(r=0.12, p<0.05)$ and marijuana use consequences ( $r=0.15, p<0.05$ ) were found. Hypothesis three, which predicted significant negative relationships between perceived risk and personal disapproval and alcohol and marijuana use and consequences, was partially supported. Significant 
negative correlation was found between perceived risk of alcohol use and alcohol use $(r=-0.22, p<0.001)$. Significant negative correlations between personal disapproval of marijuana use and marijuana $(r=-0.19, p<0.05)$ and alcohol $(r=-0.18, p<0.05)$ use were found. Significant negative correlation was found between personal disapproval of alcohol use and alcohol use $(r=-0.24, p<0.001)$.

Hypotheses four and five were analyzed via hierarchical multiple regression (Figure 1.1). Hypothesis four predicted that strength of ethnic identity would moderate the relationship between neighborhood risk, perceived risk, and personal disapproval and marijuana and alcohol use and consequences. The first set of HMR analyses focused on marijuana (Table 11) and alcohol (Table 12) use. Gender was placed in step one of the analyses to account for the potential gender differences demonstrated by ANOVAs in the preliminary analysis. Gender was not found to be a significant predictor of marijuana use. When added to the model predicting marijuana use, ethnic identity, neighborhood risk, perceived risk of marijuana use and personal disapproval of marijuana use were found to be significant predictors $F(5,279)=4.57$, $p<0.05, \mathrm{R}^{2}=0.08$. Significant medium to large effects sizes were found for ethnic identity ( $\beta=0.17)$, neighborhood risk $(\beta=-0.16)$, and personal disapproval of marijuana use $(\beta=-0.18)$. The model remained significant when interaction effects were added in step three, $F(8,271)=3.07, p<0.05, \mathrm{R}^{2}=0.08$, resulting in a significant small $0.6 \%$ change in the predictive capacity of the model. However, significant effects were not found when analyzing the individual impact of each interaction effect indicating that ethnic identity does not significantly moderate the 
impact of neighborhood risk, perceived risk, and personal disapproval on marijuana use.

Gender was not found to be a significant predictor of alcohol use. When added to the model predicting alcohol use, ethnic identity, neighborhood risk, perceived risk of alcohol use and personal disapproval of alcohol use were found to be significant predictors $F(5,279)=5.31, p<0.001, \mathrm{R}^{2}=0.09$. Significant medium to large effects sizes were found for personal disapproval of alcohol use $(\beta=-0.18)$. The model remained significant when interaction effects were added in step three, $F(8,271)=$ 3.42, $p<0.05, \mathrm{R}^{2}=0.09$, resulting in a significant small $0.3 \%$ change in the predictive capacity of the model. However, significant effects were not found when analyzing the individual impact of each interaction effect indicating that ethnic identity did not significantly moderate the impact of neighborhood risk, perceived risk, and personal disapproval on alcohol use.

The second set of HMR analyses focused on marijuana (Table 13) and alcohol (Table 14) use consequences. Gender was found to be a significant predictor of marijuana use consequences, $F(1,278)=3.351, p<0.05, \mathrm{R}^{2}=0.018$. Significant small to medium effect of gender $(\beta=0.134)$ was found. When added to the model predicting marijuana use, ethnic identity, neighborhood risk, perceived risk of marijuana use and personal disapproval of marijuana use were found to be significant predictors $F(5,274)=2.998, p<0.05, \mathrm{R}^{2}=0.052$ and resulted in a $3.4 \%$ change in the predictive capacity of the model. Significant medium to large effects sizes were found for gender $(\beta=0.155)$ and ethnic identity $(\beta=0.155)$. The model remained significant when interaction effects were added in step three, $F(8,271)=3.347, p<$ 
$0.05, \mathrm{R}^{2}=0.090$, resulting in a small $3.8 \%$ change in the predictive capacity of the model. However, significant interaction effects were only found between ethnic identity and perceived risk of marijuana use $(\beta=0.143)$.

Gender was not found to be a significant predictor of alcohol use consequences. When added to the model predicting alcohol use consequences, ethnic identity, neighborhood risk, perceived risk of alcohol use and personal disapproval of alcohol use were found to be significant predictors $F(5,275)=3.147, p<0.05, \mathrm{R}^{2}=$ 0.054. Significant small effect sizes were found for perceived risk $(\beta=-0.212)$ and personal disapproval $(\beta=0.195)$ of alcohol use. The model remained significant when interaction effects were added in step three, $F(8,271)=2.564, p<0.05, \mathrm{R}^{2}=$ 0.070 , resulting in a small $1.6 \%$ change in the predictive capacity of the model. However, significant effects were not found when analyzing the individual impact of each interaction effect indicating that ethnic identity does not significantly moderate the impact of neighborhood risk, perceived risk, and personal disapproval on alcohol use.

The final hypothesis predicted that perceived risk and personal disapproval would mediate the relationship between neighborhood risk and marijuana and alcohol use (Figure 1.2). In order to confirm mediation via hierarchical multiple regression, the variables of interest must demonstrate significant correlations with one another. Although, neighborhood risk was found to be significantly correlated with marijuana use ( $r=-0.13, p<0.05)$, significant correlations between the remaining variables were not found. Similarly, when exploring mediation with the alcohol use model, no 
significant correlations were found between the variables of interest. Because preliminary assumptions are not met, the final hypothesis is not supported. 


\section{CHAPTER 5}

\section{DISCUSSION}

Recent research has demonstrated that individuals who self-identify with two or more races may be at higher risk for adverse substance use outcomes (Chavez \& Sanchez, 2010; Choi et al., 2006a; Jackson \& LeCroy, 2009; Sakai, Wang, \& Price, 2010; Shih \& Sanchez, 2005; Udry, Li, \& Hendrickson-Smith, 2003). Despite evidence that suggests that this population may be at higher risk than their monoracial peers, limited research has explored the contributing factors to that potential risk. Exploration of factors contributing to this risk are especially important as this population continues to grow in the U.S. and it will be important to consider the unique needs of this population when considering the implementation of effective behavioral health interventions.

The current study set out to explore factors that could potentially impact the substance use outcomes of multiracial emerging adults. The current study specifically analyzed the effect of ethnic identity, neighborhood risk, perceived risk of substance use, and personal disapproval of substance use on current substance use and related consequences. While previous research explored the impact of these factors within monoracial populations, the current study aimed to address the gap in the literature in regards to the study of multiracial individuals.

The first of five hypotheses in this study posited significant positive relationships between neighborhood risk and alcohol and marijuana use and 
consequences. The hypothesis was supported and a significant negative relationship between neighborhood resources and marijuana use was found. These results demonstrate that the presence of less neighborhood resources (i.e., high neighborhood risk) was associated with higher level of marijuana use. This relationship was not found for alcohol use or marijuana and alcohol use related problems. The limited results found in this study may be attributable to the differing impact of factors measuring the neighborhood's physical environment in comparison to its social environment (Furr-Holden et al., 2015). The current study used the Neighborhood Developmental Assets measure which was composed of items less focused on physical environment and focused more closely on the social environment of the neighborhood. While economic disadvantage factors (e.g., unemployment and poverty) (Boardman, Finch, Ellison, Williams, \& Jackson, 2001) and physical environment (Furr-Holden et al., 2015) have been connected to adverse drug use outcomes, social environment continues to need further empirical support in the current research.

The second hypothesis focused on the relationship between ethnic identity on marijuana and alcohol use and consequences. Based on the results, the current study found that strong ethnic identity was associated with greater levels of reported marijuana use and experience of related consequences. Again this finding contradicted the hypothesized findings. However, past research has demonstrated that ethnic identity has been associated with higher levels of substance use for some racial/ethnic groups (Zamboanga, Raffaelli, \& Horton, 2006, Love, Yin, Codina, \& Zapata, 2006; Zamboanga, Schwartz, Jarvis, \& Van Tyne, 2009). For example, acculturation level and socialization norms have been suggested to contribute to higher 
reports of alcohol use among male Mexican American males with strong identification with Latino culture (Zamboanga et al., 2006). In a review of cultural factors, specifically historical and sociopolitical factors, that impact differential rates of alcohol use amongst varying racial groups, Castro et al (2014) found that acculturative stress is associated with increased alcohol consumption, which is particularly relevant for members of marginalized groups. Additionally, different groups have culturally defined expectations about alcohol use and definition for drunkenness that lead to variation in alcohol consumption (Castro et al., 2014). Although the current study posited that ethnic identity would have a positive effect, past research supports the possibility that the effect of ethnic identity varies according to race and ethnicity. It's possible that cultural beliefs, values, and practices shared among individuals within a cultural group promote engagement in substance use therefore leading to a positive relationship between ethnic identity and substance use outcomes.

Another possible explanation for these findings may also lie within the composition of the participant's multiracial identity. Some studies have demonstrated that differing experiences regarding racial fluidity and the impact of multiracial identity differs for groups when their racial/ethnic makeup includes marginalized racial groups (Harris \& Sims, 2002). Additional factors that have been theorized to influence the identity development of multiracial individuals include the following: experiences with racial discrimination, reference group orientation, identity negotiation across contexts, and evaluation of the social context (Miville et al., 2005). Miville and colleagues (2005) suggested that existing bi-racial and multiracial identity models should be extended to account for the aforementioned themes in the 
experiences of multiracial individuals. Additionally, while the current study uses a standardized score which aligns with more traditional ideas about integration of ethnic groups as the goal for multiracial individuals, more recent research suggests that identity integration may not be the only possible outcome for multiracial individuals (Miville et al., 2005). . This study did not explore individual levels of identities and whether participants held an integrated multiracial identity or shifted to or persistently held a monoracial identity and how that impacts their substance use outcomes. It's possible that these factors impacted the results of the current study. Mapping the current use of the MEIM-R to assess the ethnic identity of multiracial individuals on the evolving multiracial identity development models could provide further insight into the underlying mechanisms that contribute to alcohol and marijuana use within this population

The third hypothesis was partially supported by results demonstrating significant negative relationships between perceived risk of alcohol use and reported alcohol use, between personal disapproval of marijuana use and reported marijuana use, and personal disapproval of alcohol use and reported alcohol use. Consistent with previous research (Ndiaye et al., 2009), higher levels of personal disapproval contribute to lower levels of alcohol and marijuana use. Unlike in previous research (Corneille \& Belgrave, 2007; Lambert et al., 2004), only high levels of perceived risk of alcohol use contributed to lower levels of alcohol use. The differential effect of perceived risk is potentially explained by more recent research exploring the complex impact of risk appraisals on substance use intentions and behaviors. Sheeran, Harris, and Epton (2014) found through meta-analysis of recent research that risk appraisals 
can be broken down further into four elements: risk perception, anticipatory emotion (i.e., negative affect such as fear and worry that may precede making a decision), anticipated emotion (i.e., emotions like shame and guilt which are expected consequences of a decision), and perceived severity. Sheeran et al. (2014) also found, in contradiction with earlier research, that the direct effects of risk appraisals are small and the effect of the four elements is additive. Therefore, it seems more likely to uncover an effect if multiple elements of risk appraisal, and not just risk perception, are explored.

The fourth hypothesis explored the moderation effect of ethnic identity on neighborhood risk, perceived risk, and personal disapproval, but was partially supported. A significant interaction between ethnic identity and perceived risk was found when exploring the impact on marijuana use consequences. It appears that ethnic identity potentially mitigates the potentially negative impact of low perceived risk leading to lower levels of negative consequences as a result. Despite limited support for ethnic identity as a protective factor within this population, results demonstrating that the explored models had a significant impact on marijuana and alcohol use are aligned with existing research.

Lack of support for the predicted interaction effects are in line with the correlational findings of the study. Because the correlational relationships between ethnic identity and marijuana and alcohol use were not in the directions expected, it is unlikely that ethnic identity would have emerged as moderator in the predicted model. An exception appears to exist for perceived risk; however, it is notable that this was the only model in which gender was a significant predictor of the outcome (i.e., 
marijuana use consequences). An interaction effect emerged when this group difference was controlled, suggesting that other factors not considered within the context of the study could potentially be interfering with the other interaction effects explored. Various factors in addition to gender (e.g., age of onset and use over time) have been suggested to impact the developmental trajectory towards substance use (Chen \& Jacobson, 2012) and could have played a role in the present findings.

The final hypothesis aimed to provide support for the theory that perceived risk and personal disapproval are mediators in models exploring marijuana and alcohol use outcomes. Past research has provided evidence for the relationship between neighborhood risk and drug use (Ahern et al., 2008, Corneille \& Belgrave, 2007, Lambert et al., 2004), which was partially supported by the finding that neighborhood risk was significantly correlated with marijuana use. However, despite suggestions that drug beliefs mediate the relationship between neighborhood characteristics and drug use outcomes, the present study did not confirm this hypothesis. It's possible that the lack of variability within the current sample impacted the outcomes for this lane of exploration and others. The sample included a limited number of frequent and heavy users of alcohol and marijuana. Past research has been able to establish a mediation model in samples including significant numbers of chronic and heavy marijuana and alcohol users (Denhardt \& Murphy, 2013).

\section{Implications and Future Directions}

The current findings extend the present literature in important ways and also provides multiple avenues for future research and clinical practice. First, the current study has found that ethnic identity may not be a protective factor for multiracial 
emerging adults. It's possible that a strong ethnic identity could serve to increase adverse alcohol and marijuana use outcomes with this population of young adults. Further research to explore the impact of ethnic identity of substance use outcomes is needed as the available research is limited and further replication and study of samples could strengthen the findings of the current study. Additionally, the further exploration into the impact of identity integration particularly within the multiracial emerging adult population could provide more specific insight into the potential protective or adverse impact of ethnic identity within the diverse multiracial population.

Research on multiple identities has bred the concept of intersectionality and the interaction between privileged and oppressed identities. Additionally, the impact of multiple oppressed identities on the behavioral health outcomes have been explored. Intersectionality within the multiracial emerging adult population would be beneficial avenue to explore. The current findings suggest that factors outside of variables of interest could be impacting the results of the present study. Understanding the interaction between the integrated or unintegrated racial identities of multiracial individuals in conjunctions with other identity factors (e.g., ability status, gender identity, sexual orientation) could yield fruitful findings. The present study included a limited number of gender and sexual minorities and could not adequately explore this avenue but it's recommended that future researchers explore these potential factors.

It is important to note that participants in this study were allowed to selfidentify their racial and ethnic groups and answer questions according to those selfidentified labels. It's been common practice in research to have participants select 
from a pre-determined list of racial and ethnic labels; however, the current study provides some evidence that self-perceptions and self-identification may more adequately capture individual experiences and have significant impact on behavioral outcomes. The current results provide some support for the use of self-identification in research in order to capture nuances that are missed when providing pre-determined labels. Literature has gradually moved towards more fluid definitions of identity characteristics such as race and sexuality, however participants continue to have limited opportunities to self-identify within research studies. It's possible that allowing more opportunities to do so will yield impactful and important data as identity fluidity is explored further in research.

\section{Limitations}

Due to the non-experimental and cross-sectional design of the current study caution is advised in generalizing the findings to the larger multiracial emerging adult population. These findings represent a subset of the larger multiracial emerging adult population and the results have limited generalizability. The analyses used provide strength and direction of relationships and predictive capacity of variables, however, causality cannot be established due to the non-experimental design. While the variables of interest have been found to be significantly related to alcohol and marijuana use, evidence that those variables cause alcohol and marijuana use was not found.

Another study limitation is related to the measures used. The MEIM-R has been used in research with monoracial individuals; however, this is the first study to use the measure to measure multiracial identity. While the current study demonstrated 
strong reliability, further development of measures developed and standardized on multiracial population are needed and should be used. Additionally, the AUDIT-C is a measure that is often use for its clinical utility and reliability; however, it focuses specifically on measures of use that could potentially indicate hazardous use or the presence of an alcohol use disorder. A measure focused less on clinically significant problematic use may be more appropriate especially when considering the population of emerging adults.

Despite obtaining information about education level, the current study did not distinguish between emerging adults currently in college and those of the same age who are currently not in college. Important differences in regards to developmental trajectories of college students and their non-college counterparts exist and should be explored further. The inability to explore this group difference limits the potential data that could be extracted from the current sample. Lastly, more comprehensive measurement of risk appraisals would provide a more complex and inclusive assessment of the impact of risk appraisals on substance use outcomes. The current study limited its measure to one aspect of risk appraisals (i.e., risk perception) excluding other potentially confounding elements of risk appraisal.

\section{Conclusion}

The current study aimed to get a better understanding of factors that contributed to increased risk for adverse substance use outcomes amongst multiracial emerging adults. The current study particularly explored the potential impact of ethnic identity as a protective factor from increased neighborhood risk and decreased perceived risk and personal disapproval. Contrastingly, the results provided evidence 
supporting that ethnic identity may increase marijuana and alcohol use among multiracial emerging adults. Although the original hypotheses were only partially supported, the current study yielded important results that add to the limited research involving the substance use outcomes of multiracial young adults. Further research is needed to better understand the mechanisms by which ethnic identity impacts substance use outcomes amongst multiracial young adults. 


\section{APPENDICES}

Appendix A - Additional Information Regarding the Study of Ethnic Identity in Substance Use Literature

Much of what is known about ethnic identity in regards to substance use outcomes is found in research with monoracial populations. For example, research has found evidence that low ethnic identity in populations of African American and Puerto Rican adolescents and young adults are connected to reports of nicotine dependence and adult smoking (Brook, Duan, Brook, \& Ning, 2007; Brook, Zhang, Finch, \& Brook, 2010). While direct relationships between ethnic identity and substance use has limited support, research suggests that ethnic identity serves as a promotive factor through indirect relations with other intervening variables (e.g., parental monitoring) (Nagoshi, Marsiglia, Parsai, \& Castro, 2011). Ndiaye, Hecht, Wagstaff, and Elek (2009) demonstrated the potential for ethnic identity as a promotive factor because of its significant relations to personal antidrug norms, descriptive antidrug norms, refusal self-efficacy, and a decreased intention to use drugs in the future in a population of Mexican American young adults. Further support is provided by evidence, based on examination of main effects, suggesting that Afrocentric beliefs are promotive against early alcohol use initiation and lifetime alcohol use (Nasim, Belgrave, Jagers, Wilson, \& Owens, 2007).

The protective effect of ethnic identity is supported by studies that have demonstrated that ethnic identity moderates the influence of peer risk behavior on 
heavy alcohol consumption (Nasim et al., 2007), neighborhood risk on drug attitudes, sex efficacy, and intentions to use substances in the future (Corneille \& Belgrave, 2007), and substance use willingness on cigarette, alcohol and marijuana use (Stock et al., 2013). Existing research with Asian American youth contributes to the shift from viewing ethnic identity at static and stable throughout the lifetime to viewing ethnic identity as fluid and contextually linked (Hunt, Moloney, \& Evans, 2011; Moloney, Hunt, \& Evans, 2008). This research suggests that ethnic identity, at least for Asian American youth, shapes views about substance use and is closely linked to decisions to engage in substance use (Hunt, Moloney, \& Evans, 2011; Moloney, Hunt, \& Evans, 2008).

In contrast to research highlighting the potential benefits of high ethnic identity, there is research that suggests no direct or weak connections between cultural identity and substance use outcomes and risky behaviors (Baldwin, Brown, Wayment, Nez, \& Belsford, 2011). Despite finding limited evidence to support the direct link between ethnic identity and substances use, researchers have highlighted the importance of exploring the impact of cultural identity on other intervening or moderating variables related to substance use and risky behaviors. In addition to questions about direct or weak connections, research has shown that strong ethnic identity is related to greater risk for adverse health outcomes related to substance use for certain populations. For example, literature has found the higher ethnic identity is associated with a greater likelihood to use marijuana and alcohol (Zamboanga, Raffaelli, \& Horton, 2006), increased cigarette and alcohol use (Love, Yin, Codina, \& Zapata, 2006; Zamboanga, Schwartz, Jarvis, \& Van Tyne, 2009) for individuals 
descended from and who have emigrated from Spanish-speaking countries (e.g., Spain, Argentina, Colombia, Venezuela, and Mexico). 
Appendix B - Sample Advertisement

THE UNIVERSITY OF RHODE ISLAND

Department of Psychology

\title{
VOLUNTEERS WANTED FOR RESEARCH STUDY
}

\author{
Study Title: Combating neighborhood risk: The potential impact of multiracial \\ ethnic identity
}

Graduate Student Investigator: Emilie B. Joseph, M.S.

Faculty Investigator: Jasmine Mena, Ph.D.

Purpose: The purpose of this study is to explore the relationship between neighborhood risk factors, ethnic identity, drug beliefs and attitudes, and marijuana and alcohol use.

Who can participate in the study? Any individual who is 18 years old or older and identifies with more than one racial/ethnic group.

What does the study involve? This research will be conducted via an online survey which can be accessed at the link below (website listed on removal tab below).

Who do I contact if I have questions about participation in the study? If you would like further information about this study, you may contact Emilie B. Joseph, M.S. at (401)874-4100 or emilie_joseph@my.uri.edu or you may contact Jasmine Mena, Ph.D. at (401)874-2665 or jmena@uri.edu.

Additional Information: This study has been approved by the Institutional Review Board at the University of Rhode Island (IRB\# 515961-5)

Emilie Joseph, M.S.

Graduate student investigator

Jasmine Mena, $\mathrm{PhD}$

(401)874-4100

Major Professor

emilie_joseph@my.uri.edu

(401)874-2665

jmena@uri.edu

\begin{tabular}{|c|c|c|c|c|c|c|}
\hline 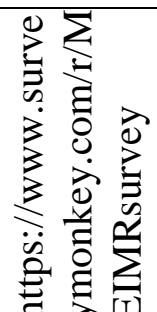 & 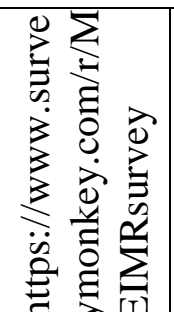 & 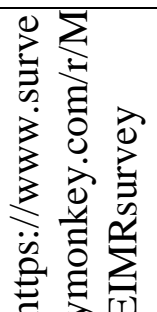 & 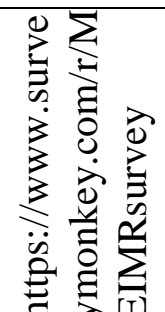 & 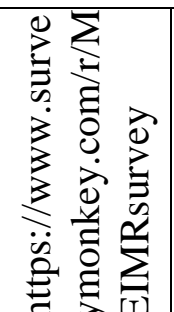 & 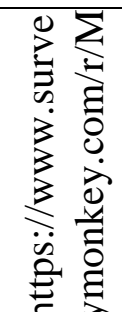 & 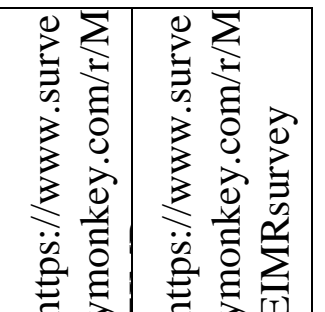 \\
\hline
\end{tabular}


Appendix C - Informed Consent

The University of Rhode Island

Department of Psychology

Chafee Hall

10 Chafee Rd

Kingston, RI 02881

Title of project: Combating neighborhood risk: The potential impact of multiracial ethnic identity

Dear Participant,

You have been invited to take part in the research project described below. If you have any questions, please feel free to contact Emilie Joseph, M.S., graduate student investigator, at (401)874-4100 or Jasmine Mena, $\mathrm{PhD}$, faculty investigator, at (401)874-2665.

Description of the project:

This study seeks to explore the relationships between neighborhood characteristics, ethnic identity, drug use beliefs and attitudes, and marijuana and alcohol use. Responses to these items will be collected and stored online through an encrypted website. Responses will then be downloaded and stored on a password protected computer. Data will be stored for three years following the completion of the study according to federal regulations. The investigator will apply for a Certificate of Confidentiality from the National Institutes of Health (NIH) to protect identifiable and sensitive information, provided by participants, from forced disclosure.

What will be done:

If you decide to take part in this study, your participation will involve completing an online survey regarding your perceptions of ethnic identity, rating of neighborhood characteristics and history of marijuana and alcohol use. Please allow approximately 25 minutes to complete the survey. At the end of the survey you may provide your email address for entrance into a raffle for a \$50 Amazon.com gift card. Course instructors may provide students with course credit for participation in this study. Students must refer to instructions provided by course instructors regarding verification of study participation.

Risks or discomfort:

The possible risks or discomforts of the study are minimal and may involve disclosure of personal and/or sensitive information.

Benefits of this study:

Although there will be no direct benefit to you for taking part in this study, the researcher may learn more about the ways in which neighborhood characteristics, 
ethnic identity, and drug use beliefs and attitudes contribute to intentions to use marijuana and alcohol.

Confidentiality:

Your participation in this study is anonymous. Your answers are private and no one else can know you participated in this study or find out your specific answers. Scientific reports will be based on group data and will not identify you as a participant in this project.

In case there is any injury to the subject:

Participation in this study is not expected to be harmful to you. If this study causes you any injury, you should write or call Emilie Joseph, M.S., graduate student investigator, at (401)874-4100 or Jasmine Mena, PhD, faculty investigator, at (401)874-2665. You may also call the office of the Vice President for Research, 70 Lower College Road, University of Rhode Island, Kingston, Rhode Island, telephone: (401) 874-4328.

Decision to quit at any time:

The decision to take part in this study is up to you. You do not have to participate. If you decide to take part in the study, you may quit at any time. You will not be penalized in any way if you do not wish to participate or quit the study before you complete this survey.

Rights and Complaints:

If you are not satisfied with the way this study is performed, you may discuss your complaints with Emilie Joseph, M.S., graduate student investigator, at (401)874-4100 or Jasmine Mena, PhD, faculty investigator, at (401)874-2665. In addition, if you have questions about your rights as a research participant, you may contact the office of the Vice President for Research, 70 Lower College Road, Suite 2, University of Rhode Island, Kingston, Rhode Island, telephone: (401) 874-4328.

You must be at least 18 years old to participate in this research study.

Any questions or concerns about the study can be directed to the following investigators:

Emilie Joseph, M.S.

Graduate student investigator

(401)874-4100

emilie_joseph@my.uri.edu
Jasmine Mena, Ph.D.

Faculty investigator

(401)874-2665

jmena@uri.edu

Please print this consent form for your records. By clicking "ACCEPT" at the bottom of this screen you are acknowledging that you have read and understand the information above and freely give your consent to participate in this research study. 
Appendix D - Intention to Use Description and Measures

Intentions to use substances in the future. Intentions to use marijuana will be measured using three items that comprise the Intention to Use Marijuana measure $(\alpha=$ .93) used by O'Callaghan \& Hannon (2003). Responses are rated on a seven-point scale ranging from extremely unlikely (-3) to extremely likely (3). Scores are obtained by summing the responses for each item. Intentions to use alcohol will be measured using the same three item measure; however, alcohol will be substituted for marijuana in each item. Reliability data will be generated for both measures in the proposed study.

\section{Intention to Use Marijuana \& Alcohol}

Rate each of these items using the following categories:

(-3) extremely unlikely, (-2) Unlikely, and (-1) Somewhat Unlikely, (0) Undecided, (1) Somewhat Likely, (2) Likely, (3) Extremely Likely

1. I would use marijuana if given some

2. I will try to use marijuana

3. I definitely intend to use marijuana

Rate each of these items using the following categories:

(-3) extremely unlikely, (-2) Unlikely, and (-1) Somewhat Unlikely, (0) Undecided, (1) Somewhat Likely, (2) Likely, (3) Extremely Likely

1. I would drink alcohol if given some

2. I will try to drink alcohol

3. I definitely intend to drink alcohol 
Appendix E - Survey: Demographic Information

Please indicate your

1. Age:

2. Gender:

$\square$ Female

$\square$ Male

3. Sexual orientation:

Heterosexual

Lesbian

Gay

\section{Queergender}

Transgender

Bisexual

$\square$ Queer

4. Highest level of education completed:

$\square$ Junior/Middle

School

$\square$ 1-year of High

School

2-years of High

School

3-years of High

School 4-years of High School

Training/Vocational School

1-year of College

2-years of College

- 3-years of College
Self-Identify:

Self-Identify:

5. Marital Status:

Single, Never Married

Divorced or Separated

Married

Widowed

Unmarried Partners 
Appendix F - Ethnic Identity Measure

\section{Multigroup Ethnic Identity Measure - Revised (MEIM-R)}

In this country, people come from a lot of different cultures and there are many different words to describe the different backgrounds or ethnic groups that people come from. Some examples of the names of ethnic groups are Hispanic, Black, AsianAmerican, Native American, Irish American, and White. These questions are about your ethnicity or your ethnic group and how you feel about it or react to it. You will answer these questions for each ethnic group with which you identify. ${ }^{1}$

Please fill in: In terms of ethnic group, I consider myself to be

Use the numbers below to indicate how much you agree or disagree with each statement.

(4) Strongly agree; (3) Agree; (2) Disagree; (1) Strongly disagree

1. I have spent time trying to find out more about my ethnic group, such as its history, traditions, and customs.

2. I have a strong sense of belonging to my own ethnic group.

3. I understand pretty well what my ethnic group membership means to me.

4. I have often done things that will help me understand my ethnic background better.

5. I have often talked to other people in order to learn more about my ethnic group.

6. I feel a strong attachment towards my own ethnic group.

7. My ethnicity is (choose all that apply):

a) White, or European American; Not Hispanic

b) Black or African American

c) Hispanic or Latino

d) American Indian or Alaska Native

e) Asian or Asian American

f) Native Hawaiian or Other Pacific Islander

g) Arab or Middle Eastern

h) Other:

8. My father's ethnicity is (choose all that apply):

a) White, Caucasian, Anglo or European American; Not Hispanic

b) Black or African American

c) Hispanic or Latino

d) American Indian or Alaska Native

e) Asian or Asian American

f) Native Hawaiian or Other Pacific Islander

g) Arab or Middle Eastern

h) Other:

9. My mother's ethnicity is (choose all that apply):

\footnotetext{
${ }^{1}$ Instructions were modified to have the measure completed for each ethnic group identified by the participants.
} 

a) White, Caucasian, Anglo or European American; Not Hispanic
b) Black or African American
c) Hispanic or Latino
d) American Indian or Alaska Native
e) Asian or Asian American
f) Native Hawaiian or Other Pacific Islander
g) Arab or Middle Eastern
h) Other: 
Appendix G - Neighborhood Risk Measure

Neighborhood Developmental Assets Scale

Below are a series of statements referring to the neighborhood in which you grew up. Tell us if you agree more or less with each one of them. Do this by circling the appropriate number for each of the alternatives we present.

How long did you live or have you lived in the neighborhood in which you grew up:

\begin{tabular}{lllllllll}
\hline & SD & D & SWD & N & SWA & A & SA \\
\hline 1. The adults in my neighborhood are & 1 & 2 & 3 & 4 & 5 & 6 & 7
\end{tabular}
concerned with the well-being of the youth.

2. People my age can find adults in my neighborhood to help solve a problem.

3. The adults in my neighborhood say $\begin{array}{lllllllll}1 & 2 & 3 & 4 & 5 & 6 & 7\end{array}$ that young people must be hard.

4. I identify with my neighborhood. $\quad \begin{array}{lllllllll}1 & 2 & 3 & 4 & 5 & 6 & 7\end{array}$

5. Adults in my neighborhood value $\quad \begin{array}{llllllll}1 & 2 & 3 & 4 & 5 & 6 & 7\end{array}$ the youth.

6. The adults in my neighborhood $\quad \begin{array}{lllllllll}1 & 2 & 3 & 4 & 5 & 6 & 7\end{array}$ reprimand us if we damage trees or public gardens.

$\begin{array}{lllllllll}\text { 7. I feel I am part of my } & 1 & 2 & 3 & 4 & 5 & 6 & 7\end{array}$ neighborhood.

8. I feel very connected to my $\quad \begin{array}{lllllllllll}1 & 2 & 3 & 4 & 5 & 6 & 7\end{array}$ neighborhood.

9. Living in my neighborhood makes $\quad \begin{array}{lllllllll} & 1 & 2 & 3 & 4 & 5 & 6 & 7\end{array}$ me feel that I am part of a community.

10. In my neighborhood, when adults $\quad \begin{array}{llllllllll} & 1 & 2 & 3 & 4 & 5 & 6 & 7\end{array}$ make decisions that affect young people, they listen to the youths' opinions.

11. In my neighborhood, there are $\quad \begin{array}{lllllllllll}1 & 2 & 3 & 4 & 5 & 6 & 7\end{array}$ people who sell drugs

12. During vacation, there are many activities for young people to have fun within my neighborhood.

13. Some of my friends are afraid to $\quad \begin{array}{lllllllllll}1 & 2 & 3 & 4 & 5 & 6 & 7\end{array}$ come to my neighborhood.

14. People in my neighborhood commit crimes and engage in other delinquent activities. 
15. The adults in my neighborhood would try to prevent young people from burning or breaking things (trash cans, etc.).

16. People of my age feel valued by adults in the neighborhood.

17. If a young person in my neighborhood tried to damage a car, an adult would try to stop him/her.

18. In my neighborhood, if you get involved in delinquency, an adult will scold you. ${ }^{2}$

19. Young people in my neighborhood have places to get together during bad weather.

20. The young people in my neighborhood can do so many things after school that they rarely get bored.

21. In my neighborhood, there are often fights between street gangs.

22. There are few neighborhoods, such as my own, where there are as many activities for young people.

Note. The above abbreviations coincide with the following rating options: $\mathrm{SD}=$ Strongly Disagree, $\mathrm{D}=$ Disagree, $\mathrm{SWD}=$ Somewhat Disagree, $\mathrm{N}=$ Neither Disagree nor Agree, SWA = Somewhat Agree, $\mathrm{A}=$ Agree and SA = Strongly Agree .

\footnotetext{
2 "Get into hooliganism" was changed to "engage in other delinquent activities" to clarify the nature of the question.
} 
Appendix H - Beliefs and Attitudes Measures

\section{Perceived Risk}

Rate each of these items using the following categories:

(0) no risk, (1) slight risk, (2) moderate risk, and (3) great risk

1. How much do you think people risk harming themselves (physically or in other ways), if they...
1a. ...try marijuana once or twice?
1b. ...smoke marijuana occasionally?
1c. ...smoke marijuana regularly?

2. How much do you think people risk harming themselves (physically or in other ways), if they...

2a. ...try one or two drinks of an alcoholic beverage (beer, wine, liquor)?

2b. ...take one or two drinks nearly every day?

2c. ...take four or five drinks nearly every day

$2 d$. ...have five or more drinks once or twice each weekend.

\section{Personal Disapproval}

Rate each of these items using the following categories:

(0) don’t disapprove, (1) disapprove, and (2) strongly disapprove.

1. Do you disapprove of people (who are 18 or older) doing each of the following?

1a. Trying marijuana once or twice

1b. Smoking marijuana occasionally

1c. Smoking marijuana regularly

2. Do you disapprove of people (who are 18 or older) doing each of the following?

2a. Trying one or two drinks of an alcoholic beverage (beer, wine, liquor)

2b. Taking one or two drinks nearly every day

2c. Taking four or five drinks nearly every day

2d. Having five or more drinks once or twice each weekend. 
Appendix I-Alcohol and Marijuana Use Measures

Alcohol Use

Directions: Select the option that best describes your answer to each question. In the table below, "drink" is defined by descriptions of beverages containing alcohol. Please use this as a guideline when answering questions. Your answers will remain confidential so please be honest. ${ }^{3}$

$$
\begin{aligned}
1 \text { DRINK }= & 1 \text { BEER (12 OUNCES) } \\
& 1 \text { WINE COOLER (12 OUNCES) } \\
& 1 \text { GLASS OF WINE (4 OUNCES) } \\
& 1 \text { SHOT OF LIQUOR (1 1/4 OUNCES) } \\
& 1 \text { MIXED DRINK }
\end{aligned}
$$

1. How often do you have a drink containing alcohol?

0) Never

1) Monthly or less

2) 24 times a month

3) 23 times a week

4) 4 or more times a week

2. How many standard drinks containing alcohol do you have on a typical day when drinking?

0) 1 or 2

1) 3 or 4

2) 5 or 6

3) 7 to 9

4) 10 or more

3. How often do you have six or more drinks on one occasion?

0) Never

1) Less than monthly

2) Monthly

3) Weekly

4) Daily or almost daily

Marijuana Use $^{4}$

Directions: Select the option that best describes your answer to each question. Your answers will remain confidential for please be honest. ${ }^{5}$

\footnotetext{
${ }^{3}$ The directions have been adapted from the original version of the AUDIT in order to suit research purposes.

${ }^{4}$ This measure was adapted from the Alcohol Use Disorders Identification Test (AUDIT).

${ }^{5}$ The directions have been adapted from the original version in order to suit research purposes.
} 
4. How often do you use marijuana?

0) Never

1) Monthly or less

2) 2 - 4 times a month

3) 2 - 3 times a week

4) 4 or more times a week

5. How many times do you use marijuana on a typical day?

0) 1 or 2

1) 3 or 4

2) 5 or 6

3) 7 to 9

4) 10 or more

6. How often do you use marijuana seven or more times in one week? ${ }^{6}$

0) Never

1) Monthly or less

2) 2 times a month

3) 3 times a month

4) 4 or more times a month

${ }^{6}$ This item, measuring heavy marijuana use, was adapted using the following definition introduced by Block \& Ghoneim (1993): seven or more times weekly. 
Appendix J - Alcohol and Marijuana-Related Problems Measures

RUTGERS ALCOHOL PROBLEM INDEX (RAPI, 18-item version)7

Different things happen to people while they are drinking ALCOHOL (USING MARIJUANA) or because of their ALCOHOL drinking (MARIJUANA USE). Several of these things are listed below. Indicate how many times each of these things happened to you WITHIN THE LAST YEAR.

Use the following code:

$0=$ None

$1=1-2$ times

$2=3-5$ times

$3=$ More than 5 times

How many times has this happened to you while you were DRINKING (USING MARIJUANA) or because of your DRINKING (MARIJUANA USE) during the last year?

1. Not able to do your homework or study for a test $\begin{array}{llll}\square & \square 1 & \square 2 & \square 3\end{array}$

2. Got into fights with other people (friends, relatives, strangers) $\begin{array}{llll}\square & \square 1 & \square 2 & \square 3\end{array}$

3. Missed out on other things because you spent too much money on alcohol (marijuana)
$\square 0$
$\square 1$
$\square 2$
3

4. Went to work or school drunk (high)
$\square 0$
$\square 1$
$\square 2$
3

5. Caused shame or embarrassment to someone
$\square 0$
1
$\square 2$
3

6. Neglected your responsibilities

$\square 0 \quad \square 1 \quad \square 2$

$\square 2 \quad \square 3$

7. Friends or relatives avoided you

$\square 0$

$\square 1$

$\square 2$

3

\footnotetext{
${ }^{7}$ The RAPI will be adapted to query for marijuana-related problems by replacing "drinking alcohol, drinking or alcohol drinking” with "using marijuana or marijuana use”, "drink” with "use marijuana”, "alcohol" with "marijuana", and "drunk" with high". The appropriate substitutions are indicated in bold print.
} 
8. Felt that you needed more alcohol (marijuana) than you used to in order to get the same effect

$\square 0 \quad \square 1 \quad \square 2 \quad \square 3$

9. Tried to control your drinking (marijuana use) (tried to drink (use marijuana) only at certain times of the day or in certain places, that is, tried to change your pattern of drinking (marijuana use))

$\begin{array}{llll}\square 0 & \square 1 & \square 2 & \square 3\end{array}$

10. Had withdrawal symptoms, that is, felt sick because you stopped or cut down on drinking (using marijuana)

$\square 0 \quad \square 1 \quad \square 2 \quad \square 3$

11. Noticed a change in your personality

$\begin{array}{llll}\square & \square 1 & \square 2 & \square 3\end{array}$

12. Felt that you had a problem with alcohol (marijuana)

$\begin{array}{llll}\square & \square 1 & \square 2 & \square 3\end{array}$

13. Missed a day (or part of a day) of school or work

$\square 0 \quad \square 1 \quad \square 2 \quad \square 3$

14. Suddenly found yourself in a place that you could not remember getting to $\begin{array}{llll}0 & \square 1 & \square 2 & \square 3\end{array}$

15. Passed out or fainted suddenly

$\begin{array}{llll}\square & \square 1 & \square 2 & \square 3\end{array}$

16. Kept drinking (using marijuana) when you promised yourself not to $\begin{array}{llll}0 & \square 1 & \square 2 & \square 3\end{array}$

17. Felt physically or psychologically dependent on alcohol (marijuana)
$\square 0$
$\square 1$
$\square 2$
$\square 3$

18. Was told by a friend, neighbor or relative to stop or cut down drinking (marijuana us)

$\square 0 \quad \square 1 \quad \square 2 \quad \square 3$ 
Table 1

Sample Characteristics

\begin{tabular}{|c|c|c|c|}
\hline & $N$ & & $N$ \\
\hline Gender & 281 & Education & 281 \\
\hline Female & 195 & 2 years of high school & 1 \\
\hline Male & 77 & 3 years of high school & 4 \\
\hline Queergender & 3 & 4 years of high school & 51 \\
\hline Transgender & 3 & Training/Vocational School & 7 \\
\hline Self-Identify & 3 & 1 year of college & 27 \\
\hline Sexual Orientation & 281 & 2 years of college & 50 \\
\hline Heterosexual & 222 & 3 years of college & 22 \\
\hline Lesbian & 6 & 4 years of college & 80 \\
\hline Gay & 10 & 5 years of college & 16 \\
\hline Bisexual & 32 & Graduate School (master’s) & 19 \\
\hline Queer & 4 & Graduate School (doctorate) & 3 \\
\hline \multirow{2}{*}{ Self-Identify } & \multirow{2}{*}{7} & Not Reported & 1 \\
\hline & & Marital Status & 281 \\
\hline Number of Ethnic Groups & 281 & Single, Never Married & 184 \\
\hline 2 Ethnic Groups & 245 & Divorced/Separated & 8 \\
\hline 3 Ethnic Groups & 30 & Married & 72 \\
\hline 4 Ethnic Groups & 3 & Unmarried Partners & 16 \\
\hline 5 Ethnic Groups & 3 & Not Reported & 1 \\
\hline
\end{tabular}


Table 2

Self-Identified Racial/Ethnic Groups

\begin{tabular}{|c|c|c|}
\hline \multicolumn{3}{|c|}{ Ethnic Group Self-Identified by Participants } \\
\hline African & European & Mexican \\
\hline African American & European Jewish & Middle Eastern \\
\hline Alaska Native & Filipino & Mixed \\
\hline American & French & Native \\
\hline American Indian & German & Native American \\
\hline Arab & German American & Nigerian \\
\hline Asian & Greek & Pacific Islander \\
\hline Asian American & Grenadian & Polish \\
\hline Austrian & Guyanese & Portuguese \\
\hline Bajan & Haitian & Puerto Rican \\
\hline Bi-racial & Hawaiian & Punjabi \\
\hline Black & Hispanic & Russian \\
\hline Black American & Hispanic/Black & Sindhi \\
\hline Black Asian & Hispanic/Mexican & Slavic \\
\hline Brazilian & Honduran & Spanish \\
\hline Canadian & Indian & South Asian \\
\hline Caribbean & Irish & Swedish \\
\hline Caucasian & Irish American & Taína \\
\hline Chinese & Italian & White \\
\hline Colombian & Jamaican American & White/American \\
\hline Cuban & Jewish & White/Caucasian \\
\hline Dravidian & Korean & \\
\hline Ecuadorian & Latina & \\
\hline El Salvadorian & Latino & \\
\hline English & Latinx & \\
\hline
\end{tabular}


Table 3

Pre-selected Racial/Ethnic Groups (Participants)

\begin{tabular}{lcccc}
\hline Pre-selected Racial/Ethnic Groups & \multicolumn{5}{c}{$\mathrm{N}$} \\
& 1 st & 2nd & 3rd & 4th \\
\hline White/European American; Not Hispanic & 182 & - & - & - \\
Black/African American & 30 & 28 & - & - \\
Hispanic/Latino & 40 & 83 & 4 & - \\
American Indian/Alaskan Native & 4 & 35 & 9 & 4 \\
Asian/Asian American & 16 & 35 & 6 & - \\
Native Hawaiian/Pacific Islander & 2 & 6 & 1 & - \\
Arab/Middle Eastern & 1 & 5 & 1 & - \\
Self-Identify & 1 & 5 & 2 & 1 \\
\hline
\end{tabular}


Table 4

Pre-selected Racial/Ethnic Groups (Parent 1)

\begin{tabular}{lccccc}
\hline Pre-selected Racial/Ethnic Groups & \multicolumn{5}{c}{$\mathrm{N}$} \\
& 1st & 2nd & 3rd & 4th \\
\hline White/European American; Not Hispanic & 140 & - & - & 1 \\
Black/African American & 34 & 4 & - & - \\
Hispanic/Latino & 58 & 23 & - & - \\
American Indian/Alaskan Native & 11 & 23 & 3 & - \\
Asian/Asian American & 31 & 6 & 1 & - \\
Native Hawaiian/Pacific Islander & 1 & 3 & 1 & - \\
Arab/Middle Eastern & 4 & - & 1 & - \\
Self-Identify & 2 & 5 & 1 & - \\
\hline
\end{tabular}


Table 5

Pre-selected Racial/Ethnic Groups (Parent 2)

\begin{tabular}{lcccc}
\hline Pre-selected Racial/Ethnic Groups & \multicolumn{5}{c}{$\mathrm{N}$} \\
& 1st & 2nd & 3rd & 4th \\
\hline $\begin{array}{l}\text { White/European American; Not } \\
\text { Hispanic }\end{array}$ & 110 & - & - & - \\
Black/African American & 41 & 6 & - & - \\
Hispanic/Latino & 69 & 23 & - & - \\
American Indian/Alaskan Native & 17 & 15 & 3 & - \\
Asian/Asian American & 31 & 2 & - & - \\
Native Hawaiian/Pacific Islander & 6 & 2 & - & - \\
Arab/Middle Eastern & 3 & 1 & - & - \\
Self-Identify & 4 & - & 3 & - \\
\hline
\end{tabular}


Table 6

Cronbach's Alphas for Scales and Subscales

\begin{tabular}{lc}
\hline Scale/Subscale & $\alpha$ \\
\hline Multigroup Ethnic Identity Measure - Revised & 0.832 \\
$\quad$ Exploration & 0.786 \\
$\quad$ Commitment & 0.760 \\
Neighborhood Developmental Assets & 0.910 \\
$\quad$ Support \& Empowerment & 0.908 \\
$\quad$ Attachment to Neighborhood & 0.944 \\
$\quad$ Security & 0.887 \\
$\quad$ Social Control & 0.873 \\
$\quad$ Youth Activities & 0.882 \\
Perceived Risk - Marijuana Use & 0.905 \\
Perceived Risk - Alcohol Use & 0.723 \\
Personal Disapproval - Marijuana Use & 0.869 \\
Personal Disapproval - Alcohol Use & 0.734 \\
Alcohol Use Disorders Identification Test - Consumption (AUDIT-C) & 0.766 \\
Modified AUDIT-C (Marijuana Consumption) & 0.848 \\
Marijuana Use Intentions & 0.971 \\
Alcohol Use Intentions & 0.950 \\
Rutgers Alcohol Problems Index (RAPI-18) & 0.970 \\
\hline & 0.980 \\
\hline Modified RAPI-18 (Marijuana Problems) & \\
\hline
\end{tabular}


Table 7

Descriptive Statistics

\begin{tabular}{lcccr}
\hline \multicolumn{1}{c}{ Variables } & $\mathrm{N}$ & Range & Mean & \multicolumn{1}{c}{$S D$} \\
\hline Age & 281 & $18-29$ & 24.30 & 3.143 \\
MEIM-R & 281 & $45.83-100$ & 76.9851 & 12.86717 \\
NDA & 281 & $40-154$ & 95.7189 & 24.11244 \\
PRMJ & 281 & $0-9$ & 3.9786 & 2.94586 \\
PRETOH & 281 & $0-12$ & 7.4555 & 2.68041 \\
PDMJ & 281 & $0-6$ & 2.1815 & 2.09740 \\
PDETOH & 281 & $0-8$ & 3.8256 & 2.19777 \\
Mod AUDIT - C & 281 & $0-12$ & 1.0783 & 2.32554 \\
Mod RAPI-18 & 281 & $0-54$ & 3.6228 & 9.27131 \\
MJ Intentions & 281 & $-9-9$ & -3.7936 & 6.28036 \\
AUDIT-C & 281 & $0-12$ & 2.5552 & 2.45196 \\
RAPI-18 & 281 & $0-54$ & 6.2883 & 10.80503 \\
ETOH Intentions & 281 & $-9-9$ & -0.0320 & 6.00616 \\
\hline
\end{tabular}

Note. The acronyms above correspond to the following measures: MEIM-R = Multigroup Ethnic Identity Measure - Revised, NDA = Neighborhood Developmental Assets, PRMJ = Perceived Risk - Marijuana Use, PRETOH = Perceived Risk Alcohol Use, PDMJ = Personal Disapproval - Marijuana Use, $\mathrm{PDETOH}=$ Personal Disapproval - Alcohol Use, Mod AUDIT-C = Modified AUDIT-C (Marijuana Consumption), Mod RAPI-18 = Modified RAPI-18 (Marijuana Problems), MJ Intentions = Marijuana Use Intentions, AUDIT-C = Alcohol Use Disorders Identification Test - Consumption (AUDIT-C), RAPI-18 = Rutgers Alcohol Problems Index (RAPI-18), ETOH Intentions = Alcohol Use Intentions 
Table 8

Descriptive Statistics (continued)

\begin{tabular}{lccccc}
\hline \multicolumn{1}{c}{ Variables } & $\mathrm{N}$ & \multicolumn{2}{c}{ Skewness } & \multicolumn{2}{c}{ Kurtosis } \\
\cline { 3 - 6 } & & Statistic & Std. Error & Statistic & Std. Error \\
\hline Age & 281 & -0.552 & 0.145 & -0.764 & 0.290 \\
MEIM-R & 281 & 0.063 & 0.148 & -0.565 & 0.295 \\
NDA & 281 & -0.298 & 0.145 & -0.521 & 0.290 \\
PRMJ & 281 & 0.186 & 0.145 & -1.174 & 0.290 \\
PRETOH & 281 & -0.549 & 0.145 & 0.257 & 0.290 \\
PDMJ & 281 & 0.516 & 0.145 & -1.075 & 0.290 \\
PDETOH & 281 & 0.091 & 0.145 & -0.609 & 0.290 \\
Mod AUDIT $-\mathrm{C}$ & 281 & 0.652 & 0.145 & 1.969 & 0.290 \\
Mod RAPI-18 & 281 & 0.926 & 0.145 & 1.206 & 0.290 \\
MJ Intentions & 281 & 0.795 & 0.145 & -.912 & 0.290 \\
AUDIT-C & 281 & 1.212 & 0.145 & 1.491 & 0.290 \\
RAPI-18 & 281 & 1.085 & 0.145 & 1.689 & 0.290 \\
ETOH Intentions & 281 & -0.250 & 0.145 & -1.250 & 0.290 \\
\hline
\end{tabular}

Note. The acronyms above correspond to the following measures: MEIM-R = Multigroup Ethnic Identity Measure - Revised, NDA = Neighborhood Developmental Assets, PRMJ = Perceived Risk - Marijuana Use, PRETOH = Perceived Risk Alcohol Use, PDMJ = Personal Disapproval - Marijuana Use, $\mathrm{PDETOH}=$ Personal Disapproval - Alcohol Use, Mod AUDIT-C = Modified AUDIT-C (Marijuana Consumption), Mod RAPI-18 = Modified RAPI-18 (Marijuana Problems), MJ Intentions = Marijuana Use Intentions, AUDIT-C = Alcohol Use Disorders Identification Test - Consumption (AUDIT-C), RAPI-18 = Rutgers Alcohol Problems Index (RAPI-18), ETOH Intentions = Alcohol Use Intentions 
Table 9

Correlations of Scales and Subscales of Interest (Pre-Imputation)

\begin{tabular}{cccccccccccccc}
\hline & 1 & 2 & 3 & 4 & 5 & 6 & 7 & 8 & 9 & 10 & 11 & 12 & 13 \\
\hline 1 & - & - & - & - & - & - & - & - & - & - & - & - & - \\
2 & 0.11 & - & - & - & - & - & - & - & - & - & - & - & - \\
3 & $0.13^{*}$ & $0.25^{*}$ & - & - & - & - & - & - & - & - & - & - & - \\
4 & 0.01 & 0.01 & 0.01 & - & - & - & - & - & - & - & - & - & - \\
5 & -0.10 & 0.03 & -0.07 & $0.42^{*}$ & - & - & - & - & - & - & - & - & - \\
6 & 0.08 & 0.05 & 0.10 & $0.61^{*}$ & $0.25^{*}$ & - & - & - & - & - & - & - & - \\
7 & -0.06 & 0.09 & -0.04 & $0.35^{*}$ & $0.55^{*}$ & $0.54^{*}$ & - & - & - & - & - & - & - \\
8 & 0.08 & $0.14^{*}$ & $-0.14^{*}$ & -0.11 & -0.00 & $-0.19^{*}$ & -0.01 & - & - & - & - & - & - \\
9 & 0.03 & $0.17 *$ & 0.04 & 0.07 & -.06 & 0.07 & 0.05 & $0.52^{*}$ & - & - & - & - & - \\
10 & 0.01 & $0.16^{*}$ & 0.00 & $-0.31^{*}$ & -0.09 & $-0.40^{*}$ & $-0.15^{*}$ & $0.60^{*}$ & $0.35^{*}$ & - & - & - & - \\
11 & $0.20^{*}$ & 0.09 & 0.11 & -0.11 & $-0.22^{*}$ & $-0.18^{*}$ & $-0.24^{*}$ & $0.42^{*}$ & $0.38^{*}$ & $0.37^{*}$ & - & - & - \\
12 & 0.09 & $0.12^{*}$ & 0.04 & 0.08 & -0.11 & 0.04 & 0.08 & $0.42^{*}$ & $0.77^{*}$ & $0.30^{*}$ & $0.50^{*}$ & - & - \\
13 & 0.03 & -0.01 & $0.19 *$ & $-0.21 *$ & $-0.18^{*}$ & $-0.29^{*}$ & $-0.38^{*}$ & 0.09 & 0.13 & $0.39^{*}$ & $0.50^{*}$ & $0.13^{*}$ & - \\
\hline
\end{tabular}

Note. ${ }^{*} p<0.05$. The numbers above correspond to the following measures: $1=$ Age, 2 = Multigroup Ethnic Identity Measure - Revised, 3 = Neighborhood Developmental Assets, 4 = Perceived Risk - Marijuana Use, 5 = Perceived Risk - Alcohol Use, $6=$ Personal Disapproval - Marijuana Use, 7 = Personal Disapproval - Alcohol Use, 8 = Modified AUDIT-C (Marijuana Consumption), 9 = Modified RAPI-18 (Marijuana Problems), 10 = Marijuana Use Intentions, 11 = Alcohol Use Disorders Identification Test - Consumption (AUDIT-C), 12 = Rutgers Alcohol Problems Index (RAPI-18), 13 = Alcohol Use Intentions 
Table 10

Correlations of the Scales and Subscales of Interest (Post-Imputation)

\begin{tabular}{|c|c|c|c|c|c|c|c|c|c|c|c|c|c|}
\hline & 1 & 2 & 3 & 4 & 5 & 6 & 7 & 8 & 9 & 10 & 11 & 12 & 13 \\
\hline 1 & - & - & - & - & - & - & - & - & - & - & - & - & - \\
\hline 2 & $0.13^{*}$ & - & - & - & - & - & - & - & - & - & - & - & - \\
\hline 3 & $0.13^{*}$ & $0.24^{*}$ & - & - & - & - & - & - & - & - & - & - & - \\
\hline 4 & 0.01 & -0.01 & 0.00 & - & - & - & - & - & - & - & - & - & - \\
\hline 5 & -0.10 & 0.02 & -0.07 & $0.43^{*}$ & - & - & - & - & - & - & - & - & - \\
\hline 6 & 0.08 & 0.04 & 0.09 & $0.61^{*}$ & $0.25^{*}$ & - & - & - & - & - & - & - & - \\
\hline 7 & -0.03 & 0.07 & -0.05 & $0.35^{*}$ & $0.56^{*}$ & $0.54^{*}$ & - & - & - & - & - & - & - \\
\hline 8 & 0.08 & $0.12^{*}$ & $-0.13^{*}$ & -0.10 & -0.00 & $-0.19^{*}$ & -0.01 & - & - & - & - & - & - \\
\hline 9 & 0.03 & $0.15^{*}$ & 0.04 & 0.07 & -0.06 & 0.07 & 0.05 & $0.52^{*}$ & - & - & - & - & - \\
\hline 10 & 0.01 & $0.16^{*}$ & -0.00 & $-0.31^{*}$ & -0.09 & $-0.40 *$ & $-0.15^{*}$ & $0.60^{*}$ & $0.35^{*}$ & - & - & - & - \\
\hline 11 & $0.20^{*}$ & 0.10 & 0.11 & -0.11 & $-0.22 *$ & $-0.18 *$ & $-0.24^{*}$ & $0.42 *$ & $0.38^{*}$ & $0.37^{*}$ & - & - & - \\
\hline 12 & 0.09 & 0.11 & 0.04 & 0.08 & -0.11 & 0.04 & 0.08 & $0.42^{*}$ & $0.77^{*}$ & $0.30^{*}$ & $0.50^{*}$ & - & - \\
\hline 13 & 0.03 & 0.00 & $0.19^{*}$ & $-0.21^{*}$ & $-0.19 *$ & $-0.29 *$ & $-0.38^{*}$ & 0.09 & $0.13^{*}$ & $0.39^{*}$ & $0.50^{*}$ & $0.13^{*}$ & - \\
\hline
\end{tabular}

Note. ${ }^{*} p<0.05$. The numbers above correspond to the following measures: $1=$ Age, 2 = Multigroup Ethnic Identity Measure - Revised, 3 = Neighborhood Developmental Assets, 4 = Perceived Risk - Marijuana Use, 5 = Perceived Risk - Alcohol Use, $6=$ Personal Disapproval - Marijuana Use, 7 = Personal Disapproval - Alcohol Use, 8 = Modified AUDIT-C (Marijuana Consumption), 9 = Modified RAPI-18 (Marijuana Problems), 10 = Marijuana Use Intentions, 11 = Alcohol Use Disorders Identification Test - Consumption (AUDIT-C), 12 = Rutgers Alcohol Problems Index (RAPI-18), 13 = Alcohol Use Intentions 
Table 11

Moderation Hierarchical Multiple Regression Results of Marijuana Use (modified AUDIT-C)

\begin{tabular}{|c|c|c|c|c|c|c|}
\hline & $\begin{array}{r}\begin{array}{r}\text { Standardized } \\
\text { Coefficient }\end{array} \\
\beta\end{array}$ & $p$ & $F$ & $d f$ & $R^{2}$ & $\Delta R^{2}$ \\
\hline Step 1 & & & 1.65 & 1,28 & 0.01 & \\
\hline Gender & 0.08 & 0.20 & & & & \\
\hline Step 2 & & & 4.57 & 5,27 & 0.08 & 0.07 \\
\hline Gender & 0.06 & 0.30 & & & & \\
\hline MEIM-R & 0.17 & 0.01 & & & & \\
\hline NDA & -0.16 & 0.01 & & & & \\
\hline PRMJ & 0.01 & 0.85 & & & & \\
\hline PDMJ & -0.18 & 0.02 & & & & \\
\hline Step 3 & & & 3.07 & 8,271 & 0.08 & 0.01 \\
\hline Gender & 0.06 & 0.31 & & & & \\
\hline MEIM-R & 0.17 & 0.01 & & & & \\
\hline NDA & -0.16 & 0.01 & & & & \\
\hline PRMJ & 0.01 & 0.90 & & & & \\
\hline PDMJ & -0.18 & 0.01 & & & & \\
\hline MEIM-R*NDA & -0.01 & 0.83 & & & & \\
\hline MEIM-R*PRMJ & 0.07 & 0.32 & & & & \\
\hline MEIM-R*PDMJ & 0.01 & 0.88 & & & & \\
\hline
\end{tabular}


Table 12

Moderation Hierarchical Multiple Regression Results of Alcohol Use (AUDIT-C)

\begin{tabular}{|c|c|c|c|c|c|c|}
\hline & $\begin{array}{l}\text { Standardized } \\
\text { Coefficient }\end{array}$ & $p$ & $F$ & $d f$ & $R^{2}$ & $\Delta R^{2}$ \\
\hline & $\beta$ & & & & & \\
\hline Step 1 & & & 0.28 & 1,278 & 0.00 & \\
\hline Gender & 0.03 & 0.60 & & & & \\
\hline Step 2 & & & 5.31 & 5,274 & 0.09 & 0.09 \\
\hline Gender & 0.01 & 0.10 & & & & \\
\hline MEIM-R & 0.10 & 0.11 & & & & \\
\hline NDA & 0.07 & 0.27 & & & & \\
\hline PRETOH & -0.12 & 0.09 & & & & \\
\hline PDETOH & -0.18 & 0.01 & & & & \\
\hline Step 3 & & & 3.42 & 8,271 & 0.09 & 0.00 \\
\hline Gender & 0.00 & 0.97 & & & & \\
\hline MEIM-R & 0.10 & 0.11 & & & & \\
\hline NDA & 0.07 & 0.30 & & & & \\
\hline PRETOH & -0.13 & 0.07 & & & & \\
\hline PDETOH & -0.19 & 0.01 & & & & \\
\hline MEIM-R*NDA & -0.02 & 0.81 & & & & \\
\hline MEIM-R*PRETOH & 0.06 & 0.39 & & & & \\
\hline MEIM-R*PDETOH & -0.01 & 0.94 & & & & \\
\hline
\end{tabular}


Table 13

Moderation Hierarchical Multiple Regression Results of Marijuana Use Consequences (modified RAPI-18)

\begin{tabular}{lcccccc}
\hline & $\begin{array}{c}\text { Standardized } \\
\text { Coefficient }\end{array}$ & $p$ & $F$ & $d f$ & $R^{2}$ & $\Delta R^{2}$ \\
\cline { 2 - 5 } Step 1 & $\beta$ & & & & & \\
Gender & 0.13 & 0.03 & & & & \\
Step 2 & & & 2.20 & 5,274 & 0.05 & 0.03 \\
Gender & 0.16 & 0.01 & & & & \\
MEIM-R & 0.16 & 0.01 & & & & \\
NDA & 0.00 & 0.95 & & & & \\
PRMJ & 0.07 & 0.35 & & & & \\
PDMJ & 0.04 & 0.60 & & & & \\
Step 3 & & & 3.35 & 8,271 & 0.09 & 0.04 \\
Gender & 0.0278 & 0.02 & \\
MEIM-R & 0.16 & 0.01 & & & & \\
NDA & 0.16 & 0.01 & & & & \\
PRMJ & -0.01 & 0.84 & & & & \\
PDMJ & 0.05 & 0.48 & & & & \\
MEIM-R*NDA & 0.02 & 0.80 & & & & \\
MEIM-R*PRMJ & 0.14 & 0.04 & & & & \\
MEIM-R*PDMJ & 0.08 & 0.30 & & & & \\
\hline
\end{tabular}


Table 14

Moderation Hierarchical Multiple Regression Results of Alcohol Use Consequences (RAPI-18)

\begin{tabular}{|c|c|c|c|c|c|c|}
\hline & $\begin{array}{c}\begin{array}{c}\text { Standardized } \\
\text { Coefficient }\end{array} \\
\beta\end{array}$ & $p$ & $F$ & $d f$ & $R^{2}$ & $\Delta R^{2}$ \\
\hline Step 1 & & & 1.35 & 1,278 & 0.01 & \\
\hline Gender & 0.07 & 0.07 & & & & \\
\hline Step 2 & & & 3.15 & 5,274 & 0.05 & 0.05 \\
\hline Gender & 0.07 & 0.26 & & & & \\
\hline MEIM-R & 0.10 & 0.10 & & & & \\
\hline NDA & 0.01 & 0.92 & & & & \\
\hline PRETOH & -0.21 & 0.00 & & & & \\
\hline PDETOH & 0.20 & 0.01 & & & & \\
\hline Step 3 & & & 2.56 & 8,271 & 0.07 & 0.02 \\
\hline Gender & 0.06 & 0.28 & & & & \\
\hline MEIM-R & 0.10 & 0.10 & & & & \\
\hline NDA & -0.02 & 0.73 & & & & \\
\hline PRMJ & -0.24 & 0.00 & & & & \\
\hline PDMJ & 0.19 & 0.01 & & & & \\
\hline MEIM-R*NDA & 0.06 & 0.30 & & & & \\
\hline MEIM-R*PRETOH & 0.11 & 0.12 & & & & \\
\hline MEIM-R*PDETOH & 0.00 & 0.96 & & & & \\
\hline
\end{tabular}


Figure 1.1. Model Displaying Expected Moderating Influence of Ethnic Identity

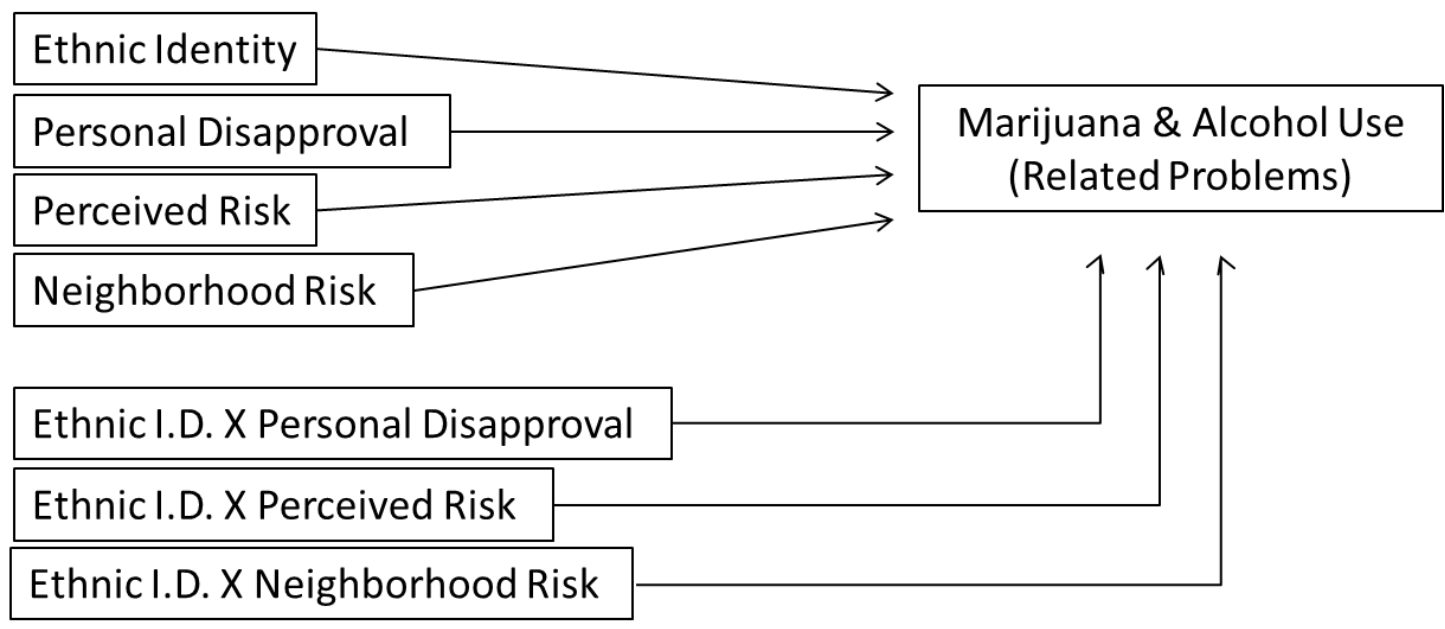

Figure 1.1. A model depicting the expected effects described in hypotheses one through four. The same model is used to predict alcohol use, marijuana-related problems, and alcohol-related problems. 
Figure 1.2. Model Displaying Mediating Effects of Personal Disapproval and Perceived Risk

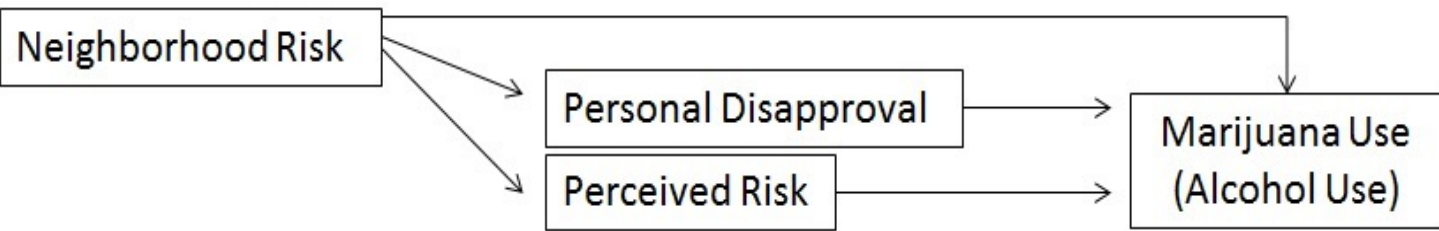

Figure 1.2. A model depicting the partial mediation described in hypothesis five. The same model is used to predict alcohol use. 


\section{BIBLIOGRAPHY}

Ahern, J., Galea, S., Hubbard, A., Midanik, L., \& Syme, S. L. (2008). Culture of drinking and individual problems with alcohol use. American Journal of Epidemiology, 167(9), 1041-1049.

Babor, T.F., Higgins-Biddle, J.C., Saunders, J.B., \& Monteiro, M.G. (2001).

AUDIT — the alcohol use disorders identification test: guidelines for use in primary care. Geneva: World Health Organization.

Baldwin, J. A., Brown, B. G., Wayment, H. A., Nez, R. A., \& Brelsford, K. M. (2011). Culture and context: Buffering the relationship between stressful life events and risky behaviors in American Indian youth. Substance Use \& Misuse, 46, $1380-1394$.

Bernard, P., Charafeddine, R., Frohlich, K.L., Daniel, M., Kestens, Y., \& Potvin, L. (2007). Health inequalities and place: A theoretical conception of neighborhood. Social Science \& Medicine, 65, 1839 - 1852.

Block, R.I. \& Ghoneim, M.M. (1993). Effects of chronic marijuana use n human cognition. Psychopharmacology, 110, 219-228.

Boardman, J. D., Finch, B. K., Ellison, C. G., Williams, D. R., \& Jackson, J. S. (2001). Neighborhood disadvantage, stress, and drug use among adults. Journal of Health and Social Behavior, 42(2), 151-165.

Brenner, A. B., Bauermeister, J. A., \& Zimmerman, M. A. (2011). Neighborhood variation in adolescent alcohol use: Examination of socioecological and social 
disorganization theories. Journal of Studies on Alcohol and Drugs, 72(4), 651659.

Brook, J. S., Duan, T., Brook, D. W., \& Ning, Y. (2007). Pathways to nicotine dependence in African American and Puerto Rican young adults. The American Journal on Addictions, 16, 450-456.

Brook, J. S., Zhang, C., Finch, S. J., \& Brook, D. W. (2010). Adolescent pathways to adult smoking: Ethnic identity, peer substance use, and antisocial behavior. The American Journal on Addictions, 19, 178 - 186.

Businelle, M. S., Kendzor, D. E., Reitzel, L. R., Costello, T. J., Cofta-Woerpel, L., Li, Wetter, D. W. (2010). Mechanisms linking socioeconomic status to smoking cessation: A structural equation modeling approach. Health Psychology, 29(3), 262-273.

Castro, F. G., Barrera, M., Mena, L. A., \& Aguirre, K. M. (2014). Culture and alcohol use. Journal of Studies on Alcohol and Drugs. Supplement, 75, 36-49.

Chao, R. K. \& Otsuki-Clutter, Michiko (2011). Racial and ethnic difference: Sociocultural and contextual explanations. Journal of Research on Adolescence, 21(1), 47-60.

Chartier, K. G., Hesselbrock, M. N., \& Hesselbrock, V. M. (2009). Ethnicity and adolescent pathways to alcohol use. Journal of Studies on Alcohol and Drugs, 70(3), 337-345.

Chauan, P. \& Widom, C. S. (2012). Childhood maltreatment and illicit drug use in middle adulthood: The role of neighborhood characteristics. Development and Psychopathology, 34, 723-738. 
Chavez, G. F. \& Sanchez, D. T. (2010). A clearer picture of multiracial Substance use: Rates and correlates of alcohol and tobacco use in multiracial adolescents and adults. Race and Social Problems, 2, 1 - 18.

Chen, H., Balan, S., \& Price, R.K. (2012). Association of contextual factors with drug use and binge drinking among White, Native American, and Mixed-Race, adolescents in the general population. Journal of Youth and Adolescence, 41(11), 1426-1441.

Chen, P., \& Jacobson, K. C. (2012). Developmental trajectories of substance use from early adolescence to young adulthood: gender and racial/ethnic differences. Journal of adolescent health, 50(2), 154-163.

Cheng, C., \& Lee, F. (2009). Multiracial Identity Integration: Perceptions of Conflict and Distance among Multiracial Individuals. Journal of Social Issues, 65(1), 51-68.

Choi, Y., Harachi, T.W., \& Catalano, R.F. (2006b). Neighborhoods, family, and substance use: Comparisons of the relations across racial and ethnic groups. Social Service Review, 80(4), 675-704.

Choi, Y., Harachi, T.W., Gillmore, M.R., \& Catalano, R.F. (2006a). Are Multiracial adolescents at greater risk? Comparison of rates, patterns, and correlates of substance use and violence between Monoracial and Multiracial adolescents. American Journal of Orthopsychiatry, 76(1), 86-97.

Choi, Y., He, M., Herrenkohl, T.L., Catalano, R.F., \& Toumbourou, J.W. (2012). Multiple identification and risks: Examination of peer factors across 
Multiracial and single-race youth. Journal of Youth and Adolescence, 41, 847 862.

Chomynova, P., Miller, P., \& Beck, F. (2009). Perceived risks of alcohol and illicit drugs: Relation to prevalence of use on individual and country level. Journal of Substance Use, 14, 250-264

Corneille, M. A. \& Belgrave, F. Z. (2007). Ethnic identity, neighborhood risk, and adolescent drug and sex attitudes and refusal efficacy: The urban African American girls’ experience. Journal of Drug Education, 37(2), 177-190.

Dennhardt, A. A., \& Murphy, J. G. (2013). Prevention and treatment of college student drug use: A review of the literature. Addictive behaviors, 38(10), 26072618.

Fagan, A. A., Wright, E. M., \& Pinchevsky, G. M. (2012). Racial/Ethnic Differences in the Relationship Between Neighborhood Disadvantage and Adolescent Substance Use. Journal of Drug Issues, 43(1), 69-84.

Furr-Holden, C. D. M., Lee, M. H., Milam, A. J., Johnson, R. M., Lee, K., \& Ialongo, N. S. (2011). The growth of neighborhood disorder and marijuana use among urban adolescents: A case for policy and environmental interventions. Journal of Studies on Alcohol and Drugs, 72(3), 371-379.

Furr-Holden, C. D. M., Lee, M. H., Johnson, R., Milam, A. J., Duncan, A., Reboussin, B. A., Leaf, P.J. \& Ialongo, N. S. (2015). Neighborhood environment and marijuana use in urban young adults. Prevention Science, 16(2), 268-278.

Gapen, M., Cross, D., Ortigo, K., Graham, A., Johnson, E., Evces, M., . . Bradley, B. (2011). Perceived neighborhood disorder, community cohesion, and PTSD 
symptoms among low-income African Americans in an urban health setting. American Journal of Orthopsychiatry, 81(1), 31-37.

Harris, D.R.\& Sim, J.J. (2002). Who is Multiracial? Assessing the complexity of live race. American Sociological Review, 67, 614 - 627.

Hawkins, J. D., Catalano, R. F., \& Miller, J. Y. (1992). Risk and protective factors for alcohol and other drug problems in adolescence and early adulthood: implications for substance abuse prevention. Psychological bulletin, 112(1), 64.

Hunt, G., Moloney, M., \& Evans, K. (2011). How Asian am I? Asian American and youth cultures, drug use, and ethnic identity construction. Youth \& Society, 43(1), $274-304$.

Jackson, K. F. \& LeCroy, C. W. (2009). The influence of race and ethnicity on substance use and negative activity involvement among monoracial and multiracial adolescents of the Southwest. Journal of Drug Education, 39(2), 195-210.

Jones-Webb, R., \& Karriker-Jaffe, K. J. (2013). Neighborhood Disadvantage, High Alcohol Content Beverage Consumption, Drinking Norms, and Drinking Consequences: A Mediation Analysis. Journal of Urban Health, 90(4), 667684

Johnston, L. D., O’Malley, P. M., Bachman, J. G., \& Schulenberg, J. E. (2012). Monitoring the Future national survey results on drug use, 1975-2011: Volume II, College students and adults ages 19-50. Ann Arbor: Institute for Social Research, The University of Michigan 
Johnston, L. D., O'Malley, P. M., Bachman, J. G., \& Schulenberg, J. E. (2013a). Monitoring the Future national results on adolescent drug use: Overview of key findings, 2012. Ann Arbor: Institute for Social Research, The University of Michigan, 1-83.

Johnston, L. D., O’Malley, P. M., Bachman, J. G., \& Schulenberg, J. E., (2013b). Monitoring the Future national survey results on drug use, 1975-2012: Volume 2, College students and adults ages 19-50. Ann Arbor: Institute for Social Research, The University of Michigan.

Johnston, L. D., O’Malley, P. M., Bachman, J. G., \& Shulenberg, J. E. (2013c). Monitoring the Future national results on drug use: 2012 Overview, Key Findings on Adolescent Drug Use. Ann Arbor: Institute for Social Research, The University of Michigan.

Karriker - Jaffe, K. J. (2013). Neighborhood socioeconomic statues and substance use by U.S. adults. Drug and Alcohol Dependence, article in press.

Kendzor, D. E., Businelle, M. S., Reitzel, L. R., Rios, D. M., Scheuermann, T. S., Pulvers, K., \& Ahluwalia, J. S. (2013). Everyday Discrimination Is Associated With Nicotine Dependence Among African American, Latino, and White Smokers. Nicotine \& Tobacco Research, 16(6), 633-640.

Kulis, S., Marsiglia, F. F., Sicotte, D., \& Nieri, T. (2007). Neighborhood effects on youth substance use in a southwestern city. Sociological Perspectives, 50(2), 273-301.

Lambert, S. F., Brown, T. L., Phillips, C. M., \& Ialongo, N. S. (2004). The relationship between perceptions of neighborhood characteristics and 
substance use among urban African American adolescents. American Journal of Community Psychology, 34, 205 - 218.

Love, A. S., Yin, Z., Codina, E., \& Zapata, J. T. (2006). Ethnic identity and risky health behaviors in school-age Mexican-American children. Psychological Reports, 98, 735-744.

Molina, K. M., Alegría, M., \& Chen, C. (2012). Neighborhood context and substance use disorders: A comparative analysis of racial and ethnic groups in the United States. Drug and Alcohol Dependence, 125, 535-543.

Moloney, M., Hunt, G., \& Evans, K. (2008). Asian American identity and drug consumption: From acculturation to normalization. Journal of Ethnicity in Substance Abuse, 7(4), 376 - 403.

Nagoshi, J. L., Marsiglia, F. F., Parsai, M., \& Castro, F. G. (2011). The moderating effects of ethnic identification on the relationship between parental monitoring and substance use in Mexican heritage adolescent in the southwest United States. Journal of Community Psychology, 39(5), 520-533.

Nasim, A., Belgrave, F. Z., Jagers, R. J., Wilson, K. D., \& Owens, K. (2007). The moderating effects of culture on peer deviance and alcohol use among highrisk African-American adolescents. Journal of Drug Education, 37(3), 335 363.

Ndiaye, K., Hecht, M., Wagstaff, D. A., \& Elek, E. (2009). Mexican-heritage preadolescents' ethnic identification and perceptions of substance use. Substance Use \& Misuse, 44, 1160- 1182. 
O’Callaghan, F.V. \& Hannon, T. (2003). Normalization of marijuana use: Its effects on adolescents’ intentions to use marijuana. Substance Use \& Misuse, 38(2), 185-199.

Oliva, A., Antolín, L., \& López, A. M. (2012). Development and validation of a scale for the measurement of adolescents' developmental assets in neighborhood. Social Indicators Research, 106(3), 563-576.

Pew Research Center. 2015. "Multiracial in America: Proud, Diverse and Growing in Numbers.” Washington, D.C.: June

Phinney, J. S. (1992). The multigroup ethnic identity measure: A new scale for use with diverse groups. Journal of Adolescent Research, 7, 156-176.

Phinney, J. S. (1996). Understanding ethnic diversity: The role of ethnic identity. American Behavioral Scientist, 40, 143 - 152.

Phinney, J. S. \& Ong, A. D. (2007). Conceptualization and measurement of ethnic identity: Current status and future directions. Journal of Counseling Psychology, 54(3), $271-281$.

Rehm, J. (2011). The Risks Associated with Alcohol Use and Alcoholism. Alcohol Res. Health, 34(2): 135-143

Saitz, R. (2005). Unhealthy alcohol use. The New England Journal of Medicine, $352,596-607$.

Sakai, J. T., Wang, C., \& Price, R. K. (2010). Substance use and dependence among Native Hawaiians, other Pacific Islanders, and Asian ethnic groups in the United States: Contrasting multiple-race and single-race prevalence rates from a national survey. Journal of Ethnicity in Substance Abuse, 9, 173-185. 
Salahuddin, N. M. \& O’Brien, K. M. (2011). Challenges and resilience in the lives of urban, multiracial adults: An instrument development study. Journal of Counseling Psychology, 58(4), 494 - 507.

Schwartz, S. J., Unger, J. B., Rosiers, S. E., Huang, S., Baezconde-Garbanati, L., Lorenzo-Blanco, E. I., . . . Szapocznik, J. (2012). Substance use and sexual behavior among recent Hispanic immigrant adolescents: Effects of parentadolescent differential acculturation and communication. Drug and Alcohol Dependence, 125.

Sheeran, P., Harris, P. R., \& Epton, T. (2014). Does heightening risk appraisals change people’s intentions and behavior? A meta-analysis of experimental studies. Psychological Bulletin, 140(2), 511.

Shih, M. \& Sanchez, D. T. (2005). Perspectives and research on the positive negative implication of having multiple racial identities. Psychological Bulletin, 131(4), 569-591.

Shih, M. \& Sanchez, D. T. (2009). When race becomes even more complex: Toward understanding the landscape of multiracial identity and experiences. Journal of Social issues, 65(1), $1-11$.

Simons, J., Correia, C.J., Carey, K.B., \& Borsari, B.E. (1998). Validating a five-factor marijuana motives measure: relations with use, problems, and alcohol motives. Journal of Counseling Psychology, 45(3), 265-273.

Stevens-Watkins, D., Perry, B., Harp, K. L., \& Oser, C. B. (2012). Racism and Illicit Drug Use Among African American Women: The Protective Effects of Ethnic 
Identity, Affirmation, and Behavior. Journal of Black Psychology, 38(4), 471496.

Stock, M. L., Gibbons, F.X., Gerard, M., Houlihan, A. E., Weng, C., Lorenz, F. O., \& Simons, R. L. (2013). Racial identification, racial composition, and substance use vulnerability among African American adolescents and young adults. Health Psychology, 35(3), 237 - 247.

Stockdale, S. E., Wells, K. B., Tang, L., Belin, T. R., Zhang, L., \& Sherbourne, C. D. (2007). The importance of social context: Neighborhood stressors, stressbuffering mechanisms, and alcohol, drug, and mental health disorders. Social Science \& Medicine, 65(9), 1867-1881.

Substance Abuse and Mental Health Services Administration, Results from the 2011 National Survey on Drug Use and Health: Summary of National Findings, NSDUH Series H-44, HHS Publication No. (SMA) 12-4713. Rockville, MD: Substance Abuse and Mental Health Services Administration, 2012.

Tucker, J. S., Pollard, M. S., de la Haye, K., Kennedy, D. P., \& Green Jr., H. D. (2013). Neighborhood characteristics and the initiation of marijuana use and binge drinking. Drug and Alcohol Dependence, 128, 83-89.

Udry, J. R., Li, R. M., \& Hendrickson-Smith, J. (2003). Health and behavior risks of adolescents with mixed-race identity. Adolescent Health, 93(11), 1865-1870.

Unger, J. B. (2012). The most critical unresolved issues associated with race, ethnicity, culture, and substance use. Substance Use \& Misuse, 47, 390-395. 
Unger, J. B, Palmer, P. H., Dent, C. W., Rohrbach, L. A., \& Johnson, C. A. (2000). Ethnic differences in adolescent smoking prevalence in California: Are multiethnic youth at higher risk? Tobacco Control, 9, ii9-ii4.

White, H. R., \& Jackson, K. (2005). Social and psychological influences on emerging adult drinking behavior. Alcohol Research \& Health, 28, 182-190.

White, H.R. \& Labouvie, E.W. (2000). Longitudinal trends in problem drinking as measured by the Rutgers Alcohol Problem Index. Alcoholism: Clinical and Experimental Research, 24, 76A.

Zamboanga, B. L., Raffaelli, M., \& Horton, N. J. (2006). Acculturation status and heavy alcohol use among Mexican American college students: Investigating the moderating role of gender. Addictive Behaviors, 31, 2188 - 2198.

Zamboanga, B. L., Schwartz, S. J., Jarvis, L. H., Van Tyne, K. (2009). Acculturation and substance use among Hispanic early adolescents: Investigating the mediating roles of acculturative stress and self-esteem. Journal of Primary Prevention, 30, 315-333. 